\title{
Soft wall holographic model for the minimal composite Higgs boson
}

\author{
Domènec Espriu $\odot^{*}$ and Alisa Katanaeva $\odot^{\dagger}$ \\ Departament de Física Quàntica i Astrofísica and Institut de Ciències del Cosmos (ICCUB), \\ Universitat de Barcelona, Martí i Franquès 1, 08028 Barcelona, Catalonia, Spain
}

(Received 17 August 2020; accepted 10 February 2021; published 11 March 2021)

\begin{abstract}
We reassess employing the holographic technique to the description of a 4D minimal composite Higgs model with $S O(5) \rightarrow S O(4)$ global symmetry breaking pattern. This particular 5D bottom-up holographic treatment is inspired by previous work in the context of QCD and it allows us to study spin one and spin zero resonances. The resulting spectrum consists of the states transforming under the unbroken $S O(4)$ subgroup and those with quantum numbers in the $S O(5) / S O(4)$ coset. The spin one states are arranged in linear radial trajectories, and the states from the broken subgroup are generally heavier. The spin zero states from the coset space correspond to the four massless Goldstone bosons in 4D. One of them takes the role of the Higgs boson. Restrictions derived from the experimental constraints (Higgs couplings, $S$ parameter, etc.) are then implemented and we conclude that the model is able to accommodate new vector resonances with masses in the range $2 \mathrm{TeV}$ to $3 \mathrm{TeV}$ without encountering phenomenological difficulties. The couplings governing the production of these new states in the processes of the Standard Model gauge boson scattering are also estimated. The method can be extended to other breaking patterns.
\end{abstract}

DOI: 10.1103/PhysRevD.103.055006

\section{INTRODUCTION}

Most of the LHC data gathered so far seems to indicate that the minimal version of the Standard Model (SM) with a doublet of complex scalar fields is compatible with the experimental results. However, many of the possible extensions involve a strongly interacting sector where perturbation theory cannot be trusted and nonperturbative methods are needed to make predictions. The extradimensional holographic framework is a valid option to investigate strongly coupled theories of various types and make meaningful comparisons with experiments.

The original AdS/CFT correspondence [1-3] between string theory on $\mathrm{AdS}_{5} \times S_{5}$ and $\mathcal{N}=4$ super Yang-Mills gauge theory on $\partial \mathrm{AdS}_{5}$ relates very particular theories on both sides. Here, we follow the bottom-up approach to holography-a conjectured phenomenological sprout of AdS/CFT that inherits several key concepts of the latter, but retains enough flexibility. It is also known as AdS/QCD due to being tried at and proven successful in describing several facets of the SM theory of strong interactions.

\footnotetext{
*espriu@icc.ub.edu

"katanaeva@fqa.ub.edu
}

Published by the American Physical Society under the terms of the Creative Commons Attribution 4.0 International license. Further distribution of this work must maintain attribution to the author(s) and the published article's title, journal citation, and DOI. Funded by SCOAP.
In the AdS/QCD models the spacetime is described by a five-dimensional anti-de Sitter (AdS) metric with the additional dimension labeled as $z$. The value $z=0$ corresponds to the ultraviolet (UV) brane, where the theory is assumed to be described by a conformal field theory (CFT) as befits QCD at short distances. In the infrared (IR) the conformality of the metric must be broken to reproduce the confining property of QCD. This could be done either via introducing an IR brane at some finite distance in the $z$-direction, or making a smooth cutoff instead. The former is known as the hard wall (HW) proposal [4,5], and the latter is called the soft wall (SW) model [6] in contrast. The SW framework is of particular phenomenological interest as it results in strongly-coupled resonances lying on linear Regge trajectories.

A viable possibility for an extended electroweak symmetry breaking sector (EWSBS) is the misaligned composite Higgs $(\mathrm{CH})$ models [7]. Characteristic to these models is the breaking of the global symmetry group $\mathcal{G}$ to a subgroup $\mathcal{H}$ due to some nonperturbative mechanism (like the condensation of the fundamental hyperfermions constructing the Higgs boson and the new resonances) at the scale $\Lambda_{\mathrm{CH}} \simeq 4 \pi f_{C H}$. The lightness of the Higgs boson is guaranteed by the identification to the Nambu-Goldstone bosons emerging after the symmetry breaking. The coset space should have capacity for at least four degrees of freedom of the Higgs doublet.

The subgroup $\mathcal{H}$ should necessarily contain $S U(2) \times$ $U(1)$. However, the SM gauge group itself lies in $\mathcal{H}^{\prime}$ which is rotated with respect to $\mathcal{H}$ by a certain angle $\theta$ around one 
of the broken directions. Vacuum misalignment, generated by a nonzero angle $\theta$, is the mechanism responsible for the electroweak (EW) breaking. Furthermore, the misalignment angle $\theta$ sets the hierarchy between $\Lambda_{\mathrm{CH}}$ and the weak scale $4 \pi v$. It is common to assume $v=f_{C H} \sin \theta$. One would expect $\sin \theta$ to be small but not too much, because a large scale separation may lead to a relevant amount of fine-tuning in order to keep light the states that should remain in the low energy part of the spectrum. Moreover, in order to naturally satisfy the constraint on the oblique parameter $T, \mathcal{H}$ should accommodate the group of custodial symmetry.

The minimal composite Higgs model (MCHM) of Ref. [8] provides the most economical way to incarnate these demands. It features the groups $\mathcal{G}=S O(5)$ and $\mathcal{H}=S O(4) \simeq S U(2) \times S U(2)$. Unfortunately, not much is known about the dynamics and the spectrum of this theory. The global symmetry $S O(5)$ cannot be realized with fermions at the microscopic level. Yet it is often implicitly assumed that a lot of qualitative features in $\mathrm{CH}$ phenomenology are similar to the ones of QCD.

There exists a substantial bibliography on the application of the holographic methods in $\mathrm{CH}$ scenarios. One way is to construct a Randall-Sundrum model on a slice of $\operatorname{AdS}\left[z_{\mathrm{UV}}, z_{\mathrm{IR}}\right]$. In this way, the minimal composite Higgs scenario was first realized in Ref. [8] (and followed in Refs. [9,10], etc.). The first example of the technique was proposed for the simplest case of the $S U(3) \rightarrow S U(2)$ breaking pattern in Ref. [11]. Other authors used flat 5D models with the $z$ dimension being an orbifold $S^{1} / Z_{2}$, i.e., restricted to a finite interval as well (see Refs. [12-14]).

The models inspired by Ref. [8] have the following characteristics. The gauge symmetry of the SM is generalized to that of $S O(5)$ and extended into the 5D bulk, where the two branes are introduced, similar to the HW option in AdS/QCD. The choice of the boundary conditions to be imposed on the 5D fields on these branes determines the symmetry breaking pattern. The Higgs boson is fully associated with the fifth component of the gauge field in the direction of the broken gauge symmetry (an idea first realized in Ref. [15]). An effective Higgs potential is absent at the tree-level, and its ColemanWeinberg generation by the quantum loop corrections (dominated by the top quark contribution) breaks the EW symmetry. Emphasis is made on the way one embeds the SM quarks into the 5D model and their impact on the said potential. EW observables $(S, T, Z \rightarrow b \bar{b})$ are also estimated [8,9].

$\mathrm{CH}$ studies have not been much elaborated in the SW framework after the initial proposal in Ref. [16]. Motivated by the much better description of QCD phenomenology that SW models provide, we would like to revisit $\mathrm{CH}$ models and provide an in-depth analysis of several relevant observables. We would like to put accent on the realization of the global symmetry breaking pattern and the description of spin zero fields, the fulfillment of the expected current algebra properties, such as Weinberg sum rules, and the operator product expansion.

Unlike the HW-like holographic $\mathrm{CH}$ setups where the $S O(5) \rightarrow S O(4)$ breaking takes part on the IR brane, in the present SW description the breaking is due to the structure of the scalar sector of the bulk Lagrangian. The skeleton is similar to generalized sigma models used for QCD at long distances [17]. The Goldstone bosons are introduced explicitly in the scalar 5D Lagrangian, but they also appear due to the gauge choice in the fifth component of the broken gauge field. The latter entry could be thought of as analogous to the gauge-Higgs construction of HW-like models, while the former is a particular feature of the SW framework. Altogether, this is reminiscent to what was proposed in Ref. [16]. However, quite differently from that paper, in our approach the dynamics responsible for the $S O(5) \rightarrow$ $S O(4)$ breaking is entirely "decoupled" from the SM gauge fields, no $S O(5)$ bulk gauge symmetry is assumed for the EW sector, and only strongly interacting composite states propagate in the bulk. It is not the case that certain boundary conditions lead to the emergence of an $S U(2) \times U(1)$ subgroup on the UV brane, but rather the gauge bosons are introduced exclusively on the boundary as external sources with the quantum numbers of $S U(2) \times U(1)$. They do not participate in the strong dynamics (except eventually through mixing of fields with identical quantum numbers) and, hence, are entirely $z$ independent.

We believe these premises to be well justified after what has been learned from holographic QCD over the last years. At least, it is worthy to take another look at $\mathrm{CH}$ models considering the amount of accumulated knowledge. To specify, our treatment is substantiated by the bottom-up holographic realizations of QCD given in Refs. [4-6,18$21]$, but several aspects of the $5 \mathrm{D}$ dynamics are quite distinct for the sake of accommodating the $\mathrm{CH}$ physics.

As we said, we concentrate on the dynamics of the strongly interacting EWSBS and its interaction with the EW sector, and no new insight into the naturalness problem or the origin of the hierarchies is provided. SM fermion introduction will be omitted in this study, though if presented they should belong exclusively to the UV brane as the EW gauge bosons do. That simultaneously closes to us the option of explaining the origin of the fermion mass hierarchy by their different placements in the bulk. It is also true that the SM fermions provide an important contribution to the radiative Higgs potential, which in its turn yields the values of $\sin \theta$, Higgs mass, and Higgs self-couplings, among other things $[22,23]$. We do, however, adopt the point of view that the Higgs potential, being of perturbative origin, is not the primary benefactor of the holographic analysis. 


\section{HOLOGRAPHIC COMPOSITE HIGGS FRAMEWORK}

\section{A. Misalignment and operators of the strongly interacting sector}

We will consider a theory where in addition to the SM $\mathcal{L}_{\mathcal{S} M}$ there is a new strongly interacting sector $\mathcal{L}_{\text {str.int. }}$, presumed to be conformal in the UV. A global symmetry of this sector is spontaneously broken following the pattern $\mathcal{G} \rightarrow \mathcal{H}$. There are Goldstone bosons in the coset space $\mathcal{G} / \mathcal{H}$, and some of them have the quantum numbers of the Higgs doublet. As the $S U(2)_{L} \times U(1)$ global group is necessarily included in $\mathcal{H}$ we can couple the EW sector of the SM to the composite sector

$$
\mathcal{L}=\tilde{\mathcal{L}}_{\text {str.int. }}+\mathcal{L}_{\mathrm{SM}}+\tilde{J}_{L}^{\alpha \mu} W_{\mu}^{\alpha}+\tilde{J}^{Y \mu} B_{\mu} .
$$

$J_{L}^{\alpha \mu}$ and $J^{Y \mu}$ are the conserved currents of the strongly interacting sector with the generators of the EW group, and only they appear in the mixing terms. Moreover, we have to denote the misalignment between the $\mathcal{H}$ subgroup of the new sector and the actual $\mathcal{H}^{\prime}$ containing the $W_{\mu}^{\alpha}$ and $B_{\mu} \mathrm{EW}$ gauge bosons. In Eq. (1), everything related to the new composite sector is marked with tildes. Let us specify the case of MCHM, where the global symmetry breaking pattern is $S O(5) \rightarrow S O(4)$ and there are exactly four Goldstones. We denote by $T^{A}, A=1, \ldots, 10$ the generators of $S O(5)$, represented by $5 \times 5$ matrices, which are traceless $\operatorname{Tr} T^{A}=0$ and are normalized as $\operatorname{Tr}\left(T^{A} T^{B}\right)=\delta^{A B}$. They separate naturally into two groups:

(i) The unbroken generators, in the case of MCHM those of $S O(4) \simeq S U(2)_{L} \times S U(2)_{R}$, we will call $T^{a}, a=1, \ldots, 6$. They are specified as

$$
T_{L}^{\alpha}=\left(\begin{array}{cc}
t_{L}^{\alpha} & 0 \\
0 & 0
\end{array}\right), \quad T_{R}^{\alpha}=\left(\begin{array}{cc}
t_{R}^{\alpha} & 0 \\
0 & 0
\end{array}\right), \quad \alpha=1,2,3,
$$

where $t_{L}^{\alpha}, t_{R}^{\alpha}$ are $4 \times 4$ matrices given by $\left(t_{L / R}^{\alpha}\right)_{j k}=$ $-\frac{i}{2}\left(\varepsilon_{\alpha \beta \gamma} \delta_{j}^{\beta} \delta_{k}^{\gamma} \pm\left(\delta_{j}^{\alpha} \delta_{k}^{4}-\delta_{k}^{\alpha} \delta_{j}^{4}\right)\right), j, k=1, \ldots, 4$.

(ii) The broken generators, corresponding to the coset $S O(5) / S O(4)$, are labeled as $\hat{T}^{i}, i=1,2,3,4$ and are defined as follows:

$$
\hat{T}_{I J}^{i}=-\frac{i}{\sqrt{2}}\left(\delta_{I}^{i} \delta_{J}^{5}-\delta_{J}^{i} \delta_{I}^{5}\right), \quad I, J=1, \ldots, 5 .
$$

A quantity parametrizing the vacuum misalignment and responsible for the EW symmetry breaking is the rotation angle $\theta$ that relates the linearly-realized global group $\mathcal{H}=$ $S O(4)$ and the gauged group $\mathcal{H}^{\prime}=S O(4)^{\prime}$. It is natural to assign the value $\theta=0$ to the SM, hence we denote the generators of $S O(5) \rightarrow S O(4)^{\prime}$ as $\left\{T^{a}(0), \hat{T}^{i}(0)\right\}$ and those of $S O(5) \rightarrow S O(4)$ as $\left\{T^{a}(\theta), \hat{T}^{i}(\theta)\right\}$. We choose a preferred direction for the misalignment and the following connection between the generators holds

$$
\begin{aligned}
T^{\alpha}(\theta) & =r(\theta) T^{\alpha}(0) r^{-1}(\theta), \quad \text { with } \\
r(\theta) & =\left(\begin{array}{ccc}
1_{3 \times 3} & 0 & 0 \\
0 & \cos \theta & \sin \theta \\
0 & -\sin \theta & \cos \theta
\end{array}\right) .
\end{aligned}
$$

Compositeness implies that some fundamental degrees of freedom are bound together by the new "color" force ("hypercolor" is usually used in the $\mathrm{CH}$ framework). MCHM does not admit complex Dirac fermions as fundamental fields at the microscopic level due to the nature of the global "flavor" symmetry group. The anomaly-free UV complete fundamental fermion theory should have $\mathcal{G}$ equivalent to $S U\left(n_{1}\right) \times \ldots \times S U\left(n_{p}\right) \times U(1)^{p-1}$, where $n_{i}$ is the number of fermions in a given irreducible representation and $p$ counts the actual number of irreducible representations [24]. The simplest UV-completable theory will be the next-to-minimal $\mathrm{CH}$ boson with $S O(6) \rightarrow S O(5)$, featuring five Goldstone bosons (other next-to-minimal patterns are mentioned, for instance, in Ref. [25]). Nevertheless, we choose to work with MCHM because of its simplicity that serves to illustrate the general procedure.

If one chooses to avoid the particularities of the microscopic structure of the new composite states (that seems advisable on the grounds of being as general as possible), it is impossible to treat the holographic MCHM completely in the AdS/QCD fashion of Ref. [4,6]. The reason is that, when constructing holographic QCD, one usually has the information on the form of the interpolating composite operators, specifically, on their scaling dimension $\Delta$. Knowing $\Delta$ is crucial to finding the particular entry in the so-called holographic dictionary $[3,26]$ that, in its turn, gives one the recipe for filling the AdS bulk with the field content. To some extent, due to affecting $\Delta$ directly, the microscopic substructure sets the prescriptions for the bulk masses and UV boundary conditions, which in their turn influence all other holographic derivations (see more on this in Sec. II C). In our holographic model describing the minimal $\mathrm{CH}$, we only use a single simple entry from this list of field-operator correspondences:

$A_{\mu}^{A}(x, z=\varepsilon)=1 \cdot \phi_{\mu}^{A}(x) \leftrightarrow \mathcal{O}_{\mu}^{A}(x) \quad$ with $\quad \Delta=3$,

where $\mathcal{O}_{\mu}^{A}(x)$ are the unspecified conserved currents of the fundamental theory containing $S O(5)$ generators $T^{A}$, and $A_{\mu}^{A}(x, z)$ are dual 5D fields restricted to provide the sources $\phi_{\mu}^{A}(x)$ for the corresponding operators on the UV brane ( $\varepsilon$ is an UV regulator). We take $\Delta=3$ (and zero bulk mass of the vector fields) as a universal feature for the conserved vector currents, because it should be so in both the case of fermionic $\left(\bar{\Psi} \gamma_{\mu} T^{A} \Psi\right)$ and bosonic $\left(\partial_{\mu} s^{\top} T^{A} s\right)$ fundamental 
degrees of freedom. The introduction of the scalar operator is indispensable in order to generate the breaking towards $S O(4)$. However, following a line similar to the vector case would mean inferring too much on the nature of the fundamental theory. Hence, we intend to construct the model so that this part of duality is realized in an alternative way.

The operators $\mathcal{O}_{\mu}^{A}(x)$ define the currents of Eq. (1):

(i) for $A=\alpha$ (left): $\frac{g}{\sqrt{2}} \mathcal{O}_{L \mu}^{\alpha}(x)=g_{V} J_{L \mu}^{\alpha}$;

(ii) for hypercharge realized as $Y=T_{R}^{3}: \frac{g^{\prime}}{\sqrt{2}} \mathcal{O}_{R \mu}^{3}(x)=$ $g_{V} J_{\mu}^{Y}$.

The coupling coefficients are not fully established because the operators are taken with an abstract normalization $g_{V}$ that will be determined to provide agreement with the common MCHM notations. The introduction of $g_{V}$ is also substantiated by the discussion in Ref. [21], where it is argued that a degree of arbitrariness in the field-operator holographic correspondence is a necessary piece of AdS/QCD constructions.

\section{B. 5D model Lagrangian}

In this subsection we put forward the details of the holographic 5D model, realizing the 4D MCHM concept. We settle upon the idea that there are two composite operators, a vector and a scalar one, that define the theory, and hence we have spin one and spin zero fields on the 5D side. These fields live in the 5D AdS bulk with a metric given by

$$
\begin{aligned}
g_{M N} d x^{M} d x^{N} & =\frac{R^{2}}{z^{2}}\left(\eta_{\mu \nu} d x^{\mu} d x^{\nu}-d^{2} z\right), \\
\eta_{\mu \nu} & =\operatorname{diag}(1,-1,-1,-1) .
\end{aligned}
$$

Holography prescribes that every global symmetry of the 4D model comes as a gauge symmetry of its 5D dual. Thus, the field dynamics is governed by the following $S O(5)$ gauge invariant action:

$$
\begin{aligned}
S_{5 \mathrm{D}}= & \frac{1}{4 g_{5}^{2}} \int d^{5} x \sqrt{-g} e^{-\Phi(z)} \operatorname{Tr} F_{M N} F_{K L} g^{M K} g^{L N} \\
& +\frac{1}{k_{s}} \int d^{5} x \sqrt{-g} e^{-\Phi(z)} \\
& \times\left[\operatorname{Tr} g^{M N}\left(D_{M} H\right)^{\top}\left(D_{N} H\right)-M_{H}^{2} \operatorname{Tr} H H^{\top}\right] .
\end{aligned}
$$

This 5D effective action includes matrix-valued scalar and vector fields and, as mentioned, is inspired by generalized sigma models used in the context of strong interactions. A similar starting action was used in the AdS/QCD study of Ref. [21]. The dimensionality of the normalization constants $g_{5}^{2}$ and $k_{s}$ is set to compensate that of the additional dimension: $\left[g_{5}^{2}\right]=\left[k_{s}\right]=E^{-1}$. To have the gravitational background of a smoothly capped off AdS spacetime we introduce a SW dilaton function $\Phi(z)=\kappa^{2} z^{2}$ in the common inverse exponent factor.

The matrix-valued field $H$ plays a cornerstone role in our construction: it collects the scalar degrees of freedom and is responsible for generating the breaking of the bulk gauge symmetries. Thus, it provides a dual description for the components of the Higgs doublet and for the global symmetry breaking on the 4D side. Let us specify its structure by putting forward elements with particular group transformations under the action of $g \in S O(5)$ and $h \in S O(4)$. The matrix $\xi$ of the Goldstone boson fields $\pi^{i}$ transforms under $S O(5)$ as: $\xi \rightarrow \xi^{\prime}=g \xi h^{\top}$. The other scalar degrees of freedom with the quantum numbers of $S O(4)$ are collected in the matrix $\Sigma$, transforming as $\Sigma \rightarrow \Sigma^{\prime}=h \Sigma h^{\top}$. The breaking from $S O(5)$ to $S O(4)$ also appears in $\Sigma$ and will be parametrized by the function $f(z)$. From these components we can construct a proper combination leading to $H \rightarrow H^{\prime}=g H g^{\top}$,

$$
\begin{aligned}
& H=\xi \Sigma \xi^{\top}, \quad \Sigma=\left(\begin{array}{cc}
0_{4 \times 4} & 0 \\
0 & f(z)
\end{array}\right)+\sigma^{a}(x, z) T^{a}, \\
& \xi=\exp \left(\frac{i \pi^{i}(x, z) \hat{T}^{i}}{\chi_{\pi}}\right),
\end{aligned}
$$

where $\left[\chi_{\pi}\right]=[f(z)]=E^{1}$. The minutiae of the scalar fields, introduced in $\Sigma$ as $\sigma^{a}$, will be further omitted in this study. It follows then that in the representation $H=H^{\top}$, the $\operatorname{Tr} H H^{\top}$ quadratic piece of Eq. (7) brings no field interactions, and the value of $M_{H}^{2}$ is of no consequence.

To make the Lagrangian invariant under the gauge transformation $\quad A_{M} \rightarrow A_{M}^{\prime}=g A_{M} g^{-1}+i g \partial_{M} g^{-1}$, the covariant derivative is introduced in the $5 \mathrm{D}$ action (7), defined as

$D_{M} H=\partial_{M} H+\left[A_{M}, H\right], \quad D_{M} H \rightarrow g D_{M} H g^{-1}$.

The field strength tensor that produces the vector field kinetic term in Eq. (7) is

$$
F_{M N}=\partial_{M} A_{N}-\partial_{N} A_{M}+\left[A_{M}, A_{N}\right]
$$

Generally, we take $A_{M}=-i A_{M}^{A} T^{A}$, where the upper index runs through both broken and unbroken indices $A_{M}^{a} T^{a}+A_{M}^{i} \hat{T}^{i}$. These $5 \mathrm{D}$ vector fields are connected to the composite spin one particles in 4D, as we will see below, and $A_{\mu}^{a}$ are generally unrelated to the $W_{\mu}^{\alpha}$ or $B_{\mu}$ gauge bosons of the EW interactions (but for their eventual mixing).

The $A_{\mu}^{A}$ fields are connected by duality to the $\mathcal{O}_{\mu}^{A}$ vector composite operators with the same generators and have the boundary condition (5). For the fifth component of the vector field we assume that 


$$
A_{z}^{A}(x, \varepsilon)=0
$$

because there is no $4 \mathrm{D}$ source for it to couple to. The common AdS/QCD gauge $A_{z}^{A} \equiv 0$ fulfills this condition trivially, but this is not the only possibility.

In the holographic $\mathrm{CH}$ models, since the study of Ref. [11], $A_{z}^{i}$ is frequently associated with the Goldstone bosons $\pi^{i}$ including the Higgs boson for $i=4$ (the socalled gauge-Higgs scenario). In the following, we are to introduce the gauge-Higgs analogue as a consequence of the gauge choice for $A_{z}^{i}$, thus augmenting the Goldstone modes that necessarily appear in $H$. Eventually, we are to discover that both sources of Goldstones are necessary for a consistent model. Though, according to the logic presented in the end of the previous subsection, the holographic dictionary entry for the field $H$ remains unspecified, and the near-boundary behavior of $\pi^{i}(x, z)$ would be determined with the help of the mentioned gauge-Higgs structure.

\section{Extraction of 4D-relevant physics}

The basic principle of AdS/CFT correspondence states that the partition function of the 4D theory and the on-shell action of its 5D holographic dual coincide in the following sense $[2,3]$ :

$$
Z_{4 \mathrm{D}}[\phi]=\left.\operatorname{Exp}_{5 \mathrm{D}}^{\text {on-shell }}\right|_{\phi(x, z) \rightarrow \phi(x, z=\varepsilon)} .
$$

Essentially, all bulk fields $\phi(x, z)$ are set to their boundary values $\phi(x, z=\varepsilon)$, which could be identified with the sources $\phi(x)$ as in the case of Eq. (5).

The dynamics of holographic fields is governed by a set of second order equations of motion (EOMs). Thus, a 5D field can be attributed with two solutions. According to the usual AdS/CFT dogma, the leading mode at small $z$ corresponds to the bulk-to-boundary propagator. It connects a source at the boundary and a value of a field in the bulk and should exhibit enough decreasing behavior in the IR region to render the right-hand side of Eq. (12) finite. The subleading mode represents an infinite series of normalizable solutions, known as the Kaluza-Klein (KK) decomposition. There, the $4 \mathrm{D}$ and $z$ dependencies are separated; the $z$ independent functions are identified with a tower of physical states at the $4 \mathrm{D}$ boundary that are further promoted into the bulk with the $z$-dependent profiles.

From consideration of the KK solutions one gets knowledge about the spectra of the composite 4D resonances while from Eq. (7), evaluated on the bulk-to-boundary solutions, one can extract the $n$-point correlation functions of the composite operators $[2,3,27]$. The 4D partition function is given by the functional integral over the fundamental fields $\varphi$ contained in the selected operators (e.g., $\mathcal{O}_{\mu}^{A}$ ) and in the fundamental Lagrangian $\mathcal{L}_{\text {str.int. }}$,
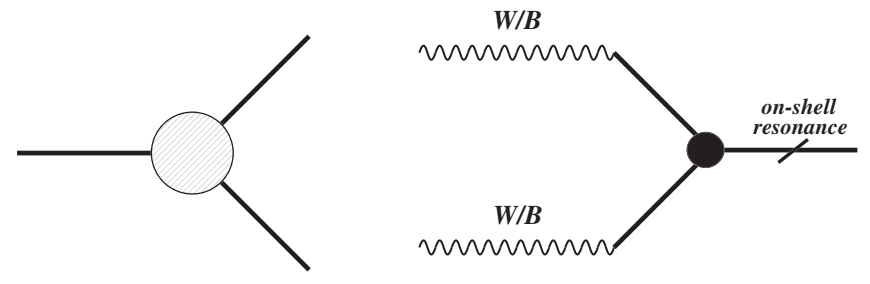

FIG. 1. Diagrams describing (left) three-point correlation function, (right) effective triple couplings between two SM gauge bosons and a composite resonance.

$$
\begin{aligned}
Z_{4 \mathcal{D}}[\phi]= & \int[\mathcal{D} \varphi] \operatorname{Exp} i \\
& \times \int d^{4} x\left[\mathcal{L}_{\text {str.int. }}(x)+\phi_{\mu}^{A}(x) \mathcal{O}^{A \mu}(x)+\ldots\right] \\
= & \operatorname{Exp} \sum_{q} \frac{1}{q !} \int \prod_{k=1}^{q} d^{4} x_{k}\left\langle\mathcal{O}_{1}\left(x_{1}\right) \ldots \mathcal{O}_{q}\left(x_{q}\right)\right\rangle \\
& \times i \phi^{1}\left(x_{1}\right) \ldots i \phi^{q}\left(x_{q}\right) .
\end{aligned}
$$

From the schematic definition in Eq. (13) and the correspondence postulate (12), it is clear that the Green's functions can be obtained by the variation of the 5D effective action with respect to the sources. Diagrammatically we can represent the correlation functions by the left panel of Fig. 1, where in general the number of legs could be equal to the number $n$ of operators in the correlator. At the same time, couplings involving just the composite resonances can be estimated by taking the proper term in the 5D Lagrangian, inserting the KK modes for the interacting 5D fields, and integrating over the $z$ dimension. Due to $\ln Z_{4 \mathrm{D}}=i S_{4 \mathrm{D}}^{\text {eff }}$, a calculation of this kind brings an effective vertex.

Interaction of a given composite state with the SM gauge bosons happens through the mixing of the latter with other composite particles. Due to the misalignment the EW bosons couple to a variety of resonances. The corresponding rotated currents $\tilde{J}_{\mu}^{\alpha}$ overlap with different types of vectorial currents that are holographically connected to vector composite fields. Besides, all radial excitations in a KK tower should generally be included in the internal propagation. The procedure in this case is the following: calculate the $n$-point correlation function, build the effective 4D Lagrangian via attaching $W_{\mu}^{\alpha}$ or $B_{\mu}$ fields as physical external sources, and reduce the legs where the composite resonances become physical and put on-shell (substituted with their KK modes). This is shown in the right panel of Fig. 1.

\section{EQUATIONS OF MOTION AND THEIR SOLUTIONS}

In this section we study the EOMs of the 5D fields. They are derived from the 5D action at the quadratic level, 


$$
\begin{aligned}
S_{5 \mathrm{D}}^{(2)}= & \int d^{5} x e^{-\Phi(z)}\left\{-\frac{1}{4 g_{5}^{2}} \frac{R}{z} F_{\mu \nu}^{A} F^{A \mu \nu}\right. \\
& +\frac{1}{2 g_{5}^{2}} \frac{R}{z}\left(\partial_{z} A_{\mu}^{A}-\partial_{\mu} A_{z}^{A}\right)\left(\partial_{z} A^{A \mu}-\partial^{\mu} A_{z}^{A}\right) \\
& \left.+\frac{f^{2}(z)}{k_{s}} \frac{R^{3}}{z^{3}}\left[\left(A_{\mu}^{i}-\partial_{\mu} \frac{\pi^{i}}{\chi_{\pi}}\right)^{2}-\left(A_{z}^{i}-\partial_{z} \frac{\pi^{i}}{\chi_{\pi}}\right)^{2}\right]\right\} .
\end{aligned}
$$

The sum over coincident indices is assumed for $A=$ $\{a, i\}=1, \ldots, 10$ in the first line, and just over broken indices $i=1, \ldots, 4$ in the second. The Ansätze functions are $\Phi(z)=\kappa^{2} z^{2}$ and $f(z) \sim z$. The choice for the symmetry breaking function $f(z)$ is justified by the analyticity of the solution in the broken vector sector; the argumentation is similar to that of Ref. [21].

\section{A. Equations of motion for the unbroken generators}

In the unbroken sector with $a=1, \ldots, 6$ the EOMs are

$$
\begin{gathered}
\partial_{z} \frac{e^{-\Phi(z)}}{z} \partial_{z} A_{\mu}^{a}-\frac{e^{-\Phi(z)}}{z} \square A_{\mu}^{a}-\partial_{z} \frac{e^{-\Phi(z)}}{z} \partial_{\mu} A_{z}^{a}=0, \\
\square A_{z}^{a}=\partial^{\mu} \partial_{z} A_{\mu}^{a} .
\end{gathered}
$$

Acting with $\partial^{\mu}$ on the first equation and substituting $\square A_{z}^{a}$ from the second one, we get the third term equal to the first one. Then, the result is

$$
\square \partial^{\mu} A_{\mu}^{a}=0,
$$

which implies the separation of solutions on transverse, $\partial^{\mu} A_{\mu}^{a}=0$, and longitudinal, $q_{A}^{2}=0$, i.e.,

$$
A_{\mu}^{a}=A_{\mu}^{a \perp}+A_{\mu}^{a \|},
$$

with $A_{\mu}^{a \perp}=\mathcal{P}_{\mu \nu} A^{a \nu}, \mathcal{P}_{\mu \nu}=\left(\eta_{\mu \nu}-\frac{q_{\mu} q_{\nu}}{q^{2}}\right)$, and $A_{\mu}^{a \|}=\frac{q_{\mu} q_{\nu}}{q^{2}} A^{a \nu}$.

The condition (17) modifies the second equation in the system into

$$
\square^{2} A_{z}^{a}=0 .
$$

While acting with $\square^{2}$ on Eq. (15), and taking into account $q_{A^{\perp}}^{2} \neq 0$, we get the following equation for the transversal mode:

$$
\partial_{z} \frac{e^{-\Phi(z)}}{z} \partial_{z} A_{\mu}^{a \perp}-\frac{e^{-\Phi(z)}}{z} \square A_{\mu}^{a \perp}=0 .
$$

However, the result for the longitudinal mode with $q_{A}^{2}=0$ turns out to be trivial, meaning that the remaining system for $A_{\mu}^{a \|}$ and $A_{z}^{a}$ is underdefined. We choose to work in a class of solutions where Eq. (19) is fulfilled with the gauge

$$
A_{z}^{a}(x, z) \equiv 0
$$

As a result, the EOM for the longitudinal mode simplifies to

$$
\partial_{z} A_{\mu}^{a \|}=0 .
$$

The following boundary terms are left in the on-shell action (14):

$$
\begin{gathered}
\left.\frac{1}{2 g_{5}^{2}} \int d^{4} x e^{-\Phi(z)} \frac{R}{z} A^{a \mu}\left(\partial_{z} A_{\mu}^{a}-\partial_{\mu} A_{z}^{a}\right)\right|_{\varepsilon} ^{\infty} \\
=-\left.\frac{1}{2 g_{5}^{2}} \int d^{4} x \frac{R}{z} A^{a \perp \mu} \partial_{z} A_{\mu}^{a \perp}\right|_{z=\varepsilon} .
\end{gathered}
$$

Only the transversal term remains, giving rise to the twopoint function studied in Sec. IVA.

Let us perform a 4D Fourier transform $A_{\mu}^{a}(x, z)=$ $\int d^{4} q e^{i q x} A_{\mu}^{a}(q, z)$ and focus on finding solutions of the EOMs. First, the transverse bulk-to-boundary propagator, which we denote $V(q, z)$, is defined by

$$
A_{\mu}^{a \perp}(q, z)=\phi_{\mu}^{a \perp}(q) \cdot V(q, z), \quad V(q, \varepsilon)=1,
$$

where $\phi_{\mu}^{a \perp}$ should be understood as a projection of the original source $\phi_{\mu}^{A \perp}=\mathcal{P}_{\mu \nu} \phi^{A \nu}$. The analogous longitudinal projection will be denoted by $\phi_{\mu}^{A \|}$.

Changing the variable in Eq. (20) to $y=\kappa^{2} z^{2}$, we arrive at the following EOM:

$$
y V^{\prime \prime}(q, y)-y V^{\prime}(q, y)+\frac{q^{2}}{4 \kappa^{2}} V(q, y)=0 .
$$

It is a particular case of the confluent hypergeometric equation (see Appendix A for a review of the properties and solutions of this equation), and the dominant mode at small $z$ is

$$
V(q, z)=\Gamma\left(-\frac{q^{2}}{4 \kappa^{2}}+1\right) \Psi\left(-\frac{q^{2}}{4 \kappa^{2}}, 0 ; \kappa^{2} z^{2}\right) .
$$

The subdominant solution [see Eq. (A3)] gives us the tower of massive states, identified with vector composite resonances at the boundary. Normalizable solutions can only be found for discrete values of the 4D momentum $q^{2}=M_{V}^{2}(n)$, and we may identify $\left.V(q, z)\right|_{q^{2}=M_{V}^{2}(n)}=$ $V_{n}(z)$. The KK decomposition is set as follows:

$$
A_{\mu}^{a \perp}(q, z)=\sum_{n=0}^{\infty} V_{n}(z) A_{\mu(n)}^{a \perp}(q) .
$$

The $z$ profile and the spectrum can be expressed using the discrete parameter $n=0,1,2, \ldots$, and 


$$
\begin{aligned}
V_{n}(z) & =\kappa^{2} z^{2} \sqrt{\frac{g_{5}^{2}}{R}} \sqrt{\frac{2}{n+1}} L_{n}^{1}\left(\kappa^{2} z^{2}\right), \\
M_{V}^{2}(n) & =4 \kappa^{2}(n+1),
\end{aligned}
$$

where $L_{n}^{m}(x)$ are the generalized Laguerre polynomials. The profiles $V_{n}(z)$ are subject to the Dirichlet boundary condition and are normalized to fulfill the orthogonality relation

$$
\frac{R}{g_{5}^{2}} \int_{0}^{\infty} d z e^{-\kappa^{2} z^{2}} z^{-1} V_{n}(z) V_{k}(z)=\delta_{n k}
$$

For the longitudinal mode, $A_{\mu}^{a \|}(q, z)$, the bulk-to-boundary solution is similarly defined. Its EOM (22), however, admits only a trivial continuation into the bulk

$$
A_{\mu}^{a \|}(q, z)=\phi_{\mu}^{a \|}(q) \cdot V^{\|}(q, z), \quad V^{\|}(q, z)=1 .
$$

The previous results are well known. Let us now see the equivalent derivation in the broken sector.

\section{B. Equations of motion for the broken generators}

The EOMs for the broken sector with $i=1, \ldots, 4$ are more complicated due to the appearance of mixing with $\pi^{i}$ :

$$
\begin{gathered}
\partial_{z} \frac{e^{-\Phi(z)}}{z}\left(\partial_{z} A_{\mu}^{i}-\partial_{\mu} A_{z}^{i}\right)-\frac{e^{-\Phi(z)}}{z} \square A_{\mu}^{i} \\
-\frac{2 g_{5}^{2} f^{2}(z) R^{2}}{k_{s}} \frac{e^{-\Phi(z)}}{z^{3}}\left(A_{\mu}^{i}-\frac{\partial_{\mu} \pi^{i}}{\chi_{\pi}}\right)=0, \\
\frac{e^{-\Phi(z)}}{z}\left(\partial^{\mu} \partial_{z} A_{\mu}^{i}-\square A_{z}^{i}\right)-\frac{2 g_{5}^{2} f^{2}(z) R^{2}}{k_{s}} \frac{e^{-\Phi(z)}}{z^{3}}\left(A_{z}^{i}-\partial_{z} \frac{\pi^{i}}{\chi_{\pi}}\right) \\
=0, \\
\partial_{z} \frac{f^{2}(z) R^{2} e^{-\Phi(z)}}{z^{3}}\left(A_{z}^{i}-\partial_{z} \frac{\pi^{i}}{\chi_{\pi}}\right) \\
-\frac{f^{2}(z) R^{2} e^{-\Phi(z)}}{z^{3}}\left(\partial^{\mu} A_{\mu}^{i}-\square \frac{\pi^{i}}{\chi_{\pi}}\right)=0
\end{gathered}
$$

Combining $\partial^{\mu} \times(31)$ with the other two equations we arrive again at the condition

$$
\square \partial^{\mu} A_{\mu}^{i}=0,
$$

with the same options $\partial^{\mu} A_{\mu}^{i}=0$ and $q_{A}^{2}=0$, as in the unbroken case. The condition on $A_{z}^{i}$ is different, though,

$$
\partial_{z} \frac{e^{-\Phi(z)}}{z} \square^{2} A_{z}^{i}-\frac{2 g_{5}^{2} f^{2}(z) R^{2} e^{-\Phi(z)}}{k_{s} z^{3}} \square^{2} \frac{\pi^{i}}{\chi_{\pi}}=0 .
$$

The system of equations obeyed by $A_{\mu}^{i \|}, A_{z}^{i}$, and $\pi^{i}$ is insufficient to determine them and we can only solve the problem with the help of an appropriate gauge condition. There are various possibilities, but we find the option explained below most useful for the physics we aspire to describe. We impose

$$
A_{z}^{i}(x, z)=\xi \partial_{z} \frac{\pi^{i}(x, z)}{\chi},
$$

where the parameter $\xi$ is arbitrary. That is reminiscent to the gauge-Higgs constructions.

The fact that $\pi^{i}(x, z)$ appears both in the scalar part of the model Lagrangian and in this gauge condition makes it distinct from other 5D fields in the model. To analyze the Goldstone solution we assume that the corresponding EOM defines the $z$-profile $\pi(x, z)$ that couples to the physical mode $\pi^{i}(x)$ on the boundary. The Neumann boundary condition, $\left.\partial_{z} \pi(x, z)\right|_{z=\varepsilon}=0$, is imposed due to Eq. (11).

Now both parts of Eq. (35) have the same $x$-dependence, and $\square^{2}$ can be taken out of the bracket. It results in the following equation on $\pi(x, z)$ :

$$
\partial_{z} \frac{e^{-\Phi(z)}}{z} \partial_{z} \pi(x, z)-\frac{2 g_{5}^{2} f^{2}(z) R^{2}}{\xi k_{s}} \frac{e^{-\Phi(z)}}{z^{3}} \pi(x, z)=0 .
$$

At the same time it allows us to get rid of $A_{z}^{i}$ and $\partial_{\mu} \frac{\pi^{i}}{\chi_{\pi}}$ in Eq. (31). Then,

$\partial_{z} \frac{e^{-\Phi(z)}}{z} \partial_{z} A_{\mu}^{i \perp}-\frac{e^{-\Phi(z)}}{z} \square A_{\mu}^{i \perp}-\frac{2 g_{5}^{2} f^{2}(z) R^{2}}{k_{s}} \frac{e^{-\Phi(z)}}{z^{3}} A_{\mu}^{i \perp}=0$,

$$
\partial_{z} \frac{e^{-\Phi(z)}}{z} \partial_{z} A_{\mu}^{i \|}-\frac{2 g_{5}^{2} f^{2}(z) R^{2}}{k_{s}} \frac{e^{-\Phi(z)}}{z^{3}} A_{\mu}^{i \|}=0 .
$$

At the boundary, we have the following terms in the effective 4D action:

$$
\begin{gathered}
\left.\int d^{4} x\left[e^{-\Phi(z)} \frac{R}{z} \frac{1}{2 g_{5}^{2}} A^{i \mu}\left(\partial_{z} A_{\mu}^{i}-\partial_{\mu} A_{z}^{i}\right)+e^{-\Phi(z)} \frac{f^{2}(z) R^{2}}{z^{3}} \frac{R}{k_{s}} \frac{\pi^{i}}{\chi_{\pi}}\left(A_{z}^{i}-\partial_{z} \frac{\pi^{i}}{\chi_{\pi}}\right)\right]\right|_{0} ^{\infty} \\
\stackrel{\xi=1}{\longrightarrow}-\left.\frac{1}{2 g_{5}^{2}} \int d^{4} x \frac{R}{z}\left(A^{i \perp \mu} \partial_{z} A_{\mu}^{i \perp}+A^{i \| \mu} \partial_{z} A_{\mu}^{i \|}-A^{i \mu} \partial_{\mu} \partial_{z} \frac{\pi^{i}}{\chi_{\pi}}\right)\right|_{z=\varepsilon}
\end{gathered}
$$


The two-point function of the longitudinal mode is nonzero and that is the crucial difference from the previous sector. The choice $\xi=1$ is explained below. For now, we observe that it makes identical the bulk EOMs for $\pi^{i}$ and $A_{\mu}^{i \|}$ and eliminates the Goldstone mass term from the boundary. For $\xi=1$, all the Goldstones (including the component associated to the Higgs boson) are massless. It is also instructive to justify the system of EOMs [Eqs. (37)-(39)] by deriving them in the model where $\xi=1$ is set from the start in Eq. (36). That exercise is worked out in Appendix B.

As in the unbroken case, we perform the 4D Fourier transform and establish the propagation between the source and the bulk for the transverse solution,

$$
A_{\mu}^{\perp}(q, z)=\phi_{\mu}^{i \perp}(q) \cdot A(q, z), \quad A(q, \varepsilon)=1 .
$$

Changing the variables to $y=\kappa^{2} z^{2}$, we arrive at the following EOM:

$y A^{\prime \prime}(q, y)-y A^{\prime}(q, y)+\left(\frac{q^{2}}{4 \kappa^{2}}-\frac{g_{5}^{2}(f(y) R)^{2}}{2 y k_{s}}\right) A(q, y)=0$.

An analytical solution of this EOM exists either for $f^{2}(y) \sim$ $y$ or $f^{2}(y) \sim$ const. The last option, taken together with the boundary condition on $A(q, z)$, leads to the implausible conclusion: $f(y)=0$. Therefore we turn to the linear Ansatz,

$$
f(z)=f \cdot \kappa z,
$$

where the constant $f$ has the dimension of mass. We also introduce a convenient parameter,

$$
a=\frac{g_{5}^{2}(f R)^{2}}{2 k_{s}} .
$$

The bulk-to-boundary mode of the confluent hypergeometric equation above is specified as

$A\left(q, \kappa^{2} z^{2}\right)=\Gamma\left(-\frac{q^{2}}{4 \kappa^{2}}+1+a\right) \Psi\left(-\frac{q^{2}}{4 \kappa^{2}}+a, 0 ; \kappa^{2} z^{2}\right)$.

The other mode for the discrete values of $q^{2}$ and $\left.A(q, z)\right|_{q^{2}=M_{A}^{2}(n)}=A_{n}(z)$ gives the $z$-profiles and the eigenstate masses

$$
\begin{aligned}
A_{n}(z) & =\kappa^{2} z^{2} \sqrt{\frac{g_{5}^{2}}{R}} \sqrt{\frac{2}{n+1}} L_{n}^{1}\left(\kappa^{2} z^{2}\right), \\
M_{A}^{2}(n) & =4 \kappa^{2}(n+1+a), \quad n=0,1,2 \ldots
\end{aligned}
$$

The orthogonality relation is completely equivalent to that of Eq. (29). In fact, the only difference is that the intercept of the Regge trajectory is larger than in the unbroken case, though the pattern is identical. These states are heavier than their unbroken counterparts, just as QCD axial vector mesons are heavier than the vector ones.

The bulk-to-boundary solution of the longitudinal EOM (39) is

$$
\begin{aligned}
A_{\mu}^{i \|}(q, z) & =\phi_{\mu}^{i \|}(q) \cdot A^{\|}(q, z), \\
A^{\|}(q, z) & =\Gamma(1+a) \Psi\left(a, 0 ; \kappa^{2} z^{2}\right) .
\end{aligned}
$$

Unlike the unbroken case, this expression is not trivial but equivalent to the transverse propagator of Eq. (46) with $q^{2}=0$.

The Goldstone EOM (37) is the same as in Eq. (39). However, $\frac{1}{2}\left(\partial_{\mu} \pi^{i}(x)\right)^{2}$ is the correct normalization of the Goldstone kinetic term in the 4D effective Lagrangian appearing after the integration over the $z$-dimension, and that fixes the constant factor differently:

$$
\pi(x, z)=F^{-1} \chi_{\pi} \Gamma(1+a) \Psi\left(a, 0 ; \kappa^{2} z^{2}\right),
$$

where

$$
F^{2}=-\frac{2 R \kappa^{2} a}{g_{5}^{2}}\left(\ln \kappa^{2} \varepsilon^{2}+2 \gamma_{E}+\psi(1+a)\right)
$$

In Sec. IVA we will find the same $F^{2}$ in the residue of the massless pole of the broken vector correlator. The exact accordance is only possible for $\xi=1$. Furthermore, solution (49) fixes the due boundary interaction:

$$
\int d^{4} x(-F) \partial^{\mu} \pi^{i}(x) \phi_{\mu}^{i}(x)
$$

As a result of $W_{\mu}^{\alpha}$ and $B_{\mu}$ couplings in Eq. (1) the mixing in Eq. (51) for $i=1,2,3$ implies that the three Goldstones would be eaten by the SM gauge bosons to provide them masses proportional to $F$. Naturally, there is no physical source to mix with the fourth Goldstone; it remains in the model as the physical Higgs particle $\pi^{4}(x)=h(x)$. The phenomenological discussion of its properties are postponed to a latter section.

To end this section, we introduce a convenient expression for the bulk-to-boundary propagators as the sums over the resonances [one should utilize Eqs. (A4) and (A6)

$$
V(q, z)=\sum_{n=0}^{\infty} \frac{F_{V}(n) V_{n}(z)}{-q^{2}+M_{V}^{2}(n)}, \quad A(q, z)=\sum_{n=0}^{\infty} \frac{F_{A}(n) A_{n}(z)}{-q^{2}+M_{A}^{2}(n)},
$$

$$
F_{A}^{2}(n)=F_{V}^{2}(n)=\frac{8 R \kappa^{4}}{g_{5}^{2}}(n+1) .
$$


Here, $F_{V / A}(n)$ are the decay constants related to the states with the corresponding quantum numbers. The longitudinal broken and Goldstone solutions could be represented by infinite sums, too.

\section{TWO-POINT CORRELATION FUNCTIONS}

\section{A. Unbroken and broken correlators}

The holographic prescriptions given in Eqs. (13) and (12) allow us to define the two-point correlation function as

$$
\begin{aligned}
\left\langle\mathcal{O}_{\mu}^{a / i}(q) \mathcal{O}_{\nu}^{b / j}(p)\right\rangle & =\delta(p+q) \int d^{4} x e^{i q x}\left\langle\mathcal{O}_{\mu}^{a / i}(x) \mathcal{O}_{\nu}^{b / j}(0)\right\rangle \\
& =\frac{\delta^{2} i S_{\text {boundary }}^{5 \mathrm{D}}}{\delta i \phi_{\mu}^{a / i}(q) \delta i \phi_{\nu}^{b / j}(p)}
\end{aligned}
$$

where the boundary remainders of the on-shell action were established in Eqs. (23) and (41). We further define the correlators:

$$
\begin{gathered}
i \int d^{4} x e^{i q x}\left\langle\mathcal{O}_{\mu}^{a / i}(x) \mathcal{O}_{\nu}^{b / j}(0)\right\rangle_{\perp} \\
=\delta^{a b / i j}\left(\frac{q_{\mu} q_{\nu}}{q^{2}}-\eta_{\mu \nu}\right) \Pi_{u n b r / b r}\left(q^{2}\right), \\
i \int d^{4} x e^{i q x}\left\langle\mathcal{O}_{\mu}^{i}(x) \mathcal{O}_{\nu}^{j}(0)\right\rangle_{\|}=\delta^{i j} \frac{q_{\mu} q_{\nu}}{q^{2}} \Pi_{b r}^{\|}\left(q^{2}\right) ;
\end{gathered}
$$

it should be taken into account that $\Pi_{\text {unbr } / b r}\left(q^{2}\right)$ are subject to short distance ambiguities of the form $C_{0}+C_{1} q^{2}$ (see, e.g., Refs. [28,29]).

Performing the due variation in Eq. (23) we find $\Pi_{u n b r}\left(q^{2}\right)$ to be

$$
\Pi_{u n b r}\left(q^{2}\right)=\left.\frac{R}{g_{5}^{2}}\left[\frac{e^{-\Phi(z)} V(q, z) \partial_{z} V(q, z)}{z}\right]\right|_{z=\varepsilon} .
$$

Let us substitute the propagator from Eq. (26), then

$$
\Pi_{u n b r}\left(q^{2}\right)=-\frac{R}{2 g_{5}^{2}} q^{2}\left(\ln \kappa^{2} \varepsilon^{2}+2 \gamma_{E}+\psi\left(-\frac{q^{2}}{4 \kappa^{2}}+1\right)\right),
$$

where $\gamma_{E}$ is the Euler-Mascheroni constant and $\psi$ is the digamma function.

To separate the short distance ambiguities we perform a decomposition of the digamma function [see Eq. (C2)] in Eq. (58),

$$
\begin{aligned}
\Pi_{u n b r}\left(q^{2}\right)= & -\frac{R}{2 g_{5}^{2}}\left(\ln \kappa^{2} \varepsilon^{2}+\gamma_{E}\right) q^{2} \\
& -\frac{2 \kappa^{2} R}{g_{5}^{2}} \sum_{n=0}^{\infty} \frac{q^{4}}{M_{V}^{2}(n)\left(q^{2}-M_{V}^{2}(n)\right)} .
\end{aligned}
$$

The first term would correspond to the ambiguity parametrizing constant $C_{1}$, while the second term is a well convergent sum over the resonances.

An alternative procedure, introducing the resonances at an earlier stage with the use of the bulk-to-boundary propagator (52) should result in the same two-point function. Taking into account the orthogonality relation (29) we get from Eq. (57),

$$
\Pi_{u n b r}\left(q^{2}\right)=\sum_{n=0}^{\infty} \frac{F_{V}^{2}(n)}{-q^{2}+M_{V}^{2}(n)} .
$$

The ambiguities appear as follows:

$$
\begin{aligned}
\Pi_{u n b r}\left(q^{2}\right)= & -\frac{2 \kappa^{2} R}{g_{5}^{2}} \sum_{n} \frac{q^{4}}{M_{V}^{2}(n)\left(q^{2}-M_{V}^{2}(n)\right)} \\
& +q^{2} \sum_{n} \frac{2 \kappa^{2} R / g_{5}^{2}}{M_{V}^{2}(n)}+\sum_{n} \frac{2 \kappa^{2} R}{g_{5}^{2}} .
\end{aligned}
$$

After the proper subtractions, we are left with the first sum of Eq. (61). This is the part relevant for the resonance description of the two-point function that coincides with the sum in Eq. (59). Hence, the convergent correlator is

$$
\hat{\Pi}_{u n b r}\left(q^{2}\right)=\sum_{n=0}^{\infty} \frac{q^{4} F_{V}^{2}(n)}{M_{V}^{4}(n)\left(-q^{2}+M_{V}^{2}(n)\right)} .
$$

Concerning the subtractions, it is not surprising that they differ for the correlators derived in two different ways. It is fundamental that they are limited to the form $C_{0}+C_{1} q^{2}$, but any reordering of the manipulations may affect the results, as this is a divergent and ill defined at short distances quantity. However, it is interesting to match the two expressions of $C_{1}$ in the proportional to $q^{2}$ terms of Eqs. (61) and (59). To do that we need to introduce a regulator in the "resonance" representation- a finite number of terms in the sum, a bound at some $N_{\max }$. Then, a connection between the maximum number of resonances, $N_{\text {max }}$, and the UV regulator $\varepsilon$ is

$$
\log N_{\max }=-2 \gamma_{E}-\log \kappa^{2} \varepsilon^{2}
$$

This relation is meaningful only at the leading order (i.e., the constant nonlogarithmic part cannot be determined by this type of heuristic argument). Finally, the last sum in Eq. (61) behaves as $\sim N_{\max }^{2}$ if we sum up a finite number of resonances, and actually corresponds to a potentially subleading logarithmic divergence. Therefore, it can be eliminated by setting the subtraction constant $C_{0}$.

In the broken vector sector the situation is very similar. For the transverse modes, the variation of Eq. (41) results in 


$$
\Pi_{b r}\left(q^{2}\right)=\left.\frac{R}{g_{5}^{2}}\left[\frac{e^{-\Phi(z)} A(q, z) \partial_{z} A(q, z)}{z}\right]\right|_{z=\varepsilon} .
$$

Substituting the propagator from Eq. (46) leads to

$$
\begin{aligned}
\Pi_{b r}\left(q^{2}\right)= & -\frac{R}{2 g_{5}^{2}} q^{2}\left(1-\frac{4 \kappa^{2} a}{q^{2}}\right) \\
& \times\left(\ln \kappa^{2} \varepsilon^{2}+2 \gamma_{E}+\psi\left(-\frac{q^{2}}{4 \kappa^{2}}+1+a\right)\right) .
\end{aligned}
$$

An alternative expression for the two-point correlator is

$$
\Pi_{b r}\left(q^{2}\right)=\sum_{n=0}^{\infty} \frac{F_{A}^{2}(n)}{-q^{2}+M_{A}^{2}(n)} .
$$

In both cases the subtraction of short distance ambiguities leads to

$$
\hat{\Pi}_{b r}\left(q^{2}\right)=\sum_{n} \frac{q^{4} F_{A}^{2}(n)}{M_{A}^{4}(n)\left(-q^{2}+M_{A}^{2}(n)\right)}-F^{2} .
$$

Here, we observe a massless pole accompanied by the decay constant $F$ anticipated in Eq. (50) and derived there from a completely different argument. Following that line, we can connect the pole to the presence of the massless Goldstone Higgs states and call it a "pion" pole, noticing the similarity to the $q^{2}=0$ pole of the axial-vector QCD correlator. It could also be expressed in the form of an infinite series,

$$
F^{2}=\frac{2 R \kappa^{2} a}{g_{5}^{2}} \sum_{n} \frac{1}{n+1+a}
$$

Variation over the longitudinal modes in Eq. (41) also brings this constant:

$$
\Pi_{b r}^{\|}\left(q^{2}\right)=F^{2} .
$$

Once more, fulfilling relation (63) makes an accordance between the order- $q^{2}$ subtractions. This demonstrates the ultraviolet origin of the renormalization ambiguity involved in the constant $C_{1}$ because the outcome is independent on whether we treat the broken or unbroken symmetries. The same could be implied about $C_{0}$. Then, the determination of $F^{2}$ in (68) is straightforward as soon as we subtract the "quadratic" term $\sum_{n} \frac{2 \kappa^{2} R}{g_{5}^{2}}$.

In the end, these correlation functions appear in the 4D effective Lagrangian as

$$
\mathcal{L}_{\text {eff }} \supset \frac{1}{2} \phi_{\mu}^{a}\left(\frac{q_{\mu} q_{\nu}}{q^{2}}-\eta_{\mu \nu}\right) \Pi_{u n b r} \phi_{\nu}^{a}+\frac{1}{2} \phi_{\mu}^{i}\left(\left(\frac{q_{\mu} q_{\nu}}{q^{2}}-\eta_{\mu \nu}\right) \Pi_{b r}+\frac{F^{2} q^{\mu} q^{\nu}}{q^{2}}\right) \phi_{\nu}^{i}
$$

\section{B. Vacuum polarization amplitudes of the gauge fields}

We started the discussion about the holographic $\mathrm{CH}$ model assuming that the SM gauge fields couple to the currents of the strongly interacting sector $\tilde{J}_{L \mu}^{\alpha}$ and $\tilde{J}_{R \mu}^{3}$ as in Eq. (1). These currents are proportional to the ones dual to the 5D fields, $\mathcal{O}_{\mu}^{a / i}$, with the EW couplings $g$ and $g^{\prime}$ necessarily appearing. We introduced the factor $g_{V}$ to modulate that proportionality. The misalignment should also be taken into account. In the notation of Eq. (4), a rotated operator can be given in terms of the original ones as $(\alpha, i=1,2,3$ here $)$

$$
\tilde{\mathcal{O}}_{L / R \mu}^{\alpha}=\frac{1 \pm \cos \theta}{2} \mathcal{O}_{L \mu}^{\alpha}+\frac{1 \mp \cos \theta}{2} \mathcal{O}_{R \mu}^{\alpha} \mp \frac{\sin \theta}{\sqrt{2}} \mathcal{O}_{\mu}^{i} .
$$

The two-point correlators of physical interest are

$$
\begin{gathered}
i \int d^{4} x e^{i q x}\left\langle\tilde{J}_{L \mu}^{\alpha}(x) \tilde{J}_{L \nu}^{\beta}(0)\right\rangle=\delta^{\alpha \beta} \frac{g^{2}}{2}\left[\left(\frac{q_{\mu} q_{\nu}}{q^{2}}-\eta_{\mu \nu}\right) \Pi_{L L}\left(q^{2}\right)+\frac{q_{\mu} q_{\nu}}{q^{2}} \Pi_{L L}^{\|}\left(q^{2}\right)\right], \\
i \int d^{4} x e^{i q x}\left\langle\tilde{J}_{R \mu}^{\alpha}(x) \tilde{J}_{R \nu}^{\beta}(0)\right\rangle=\delta^{\alpha \beta} \frac{g^{\prime 2}}{2}\left[\left(\frac{q_{\mu} q_{\nu}}{q^{2}}-\eta_{\mu \nu}\right) \Pi_{R R}\left(q^{2}\right)+\frac{q_{\mu} q_{\nu}}{q^{2}} \Pi_{R R}^{\|}\left(q^{2}\right)\right], \\
2 i \int d^{4} x e^{i q x}\left\langle\tilde{J}_{L \mu}^{\alpha}(x) \tilde{J}_{R \nu}^{\beta}(0)\right\rangle=\delta^{\alpha \beta} \frac{g g^{\prime}}{2}\left[\left(\frac{q_{\mu} q_{\nu}}{q^{2}}-\eta_{\mu \nu}\right) \Pi_{L R}\left(q^{2}\right)+\frac{q_{\mu} q_{\nu}}{q^{2}} \Pi_{L R}^{\|}\left(q^{2}\right)\right],
\end{gathered}
$$


where we defined the quantities

$$
\begin{gathered}
\Pi_{L L}\left(q^{2}\right)=\Pi_{R R}\left(q^{2}\right)=\frac{1+\cos ^{2} \theta}{2 g_{V}^{2}} \Pi_{u n b r}\left(q^{2}\right)+\frac{\sin ^{2} \theta}{2 g_{V}^{2}} \Pi_{b r}\left(q^{2}\right) \equiv \Pi_{\mathrm{diag}}\left(q^{2}\right), \\
\Pi_{L R}\left(q^{2}\right)=\frac{\sin ^{2} \theta}{g_{V}^{2}}\left(\Pi_{u n b r}\left(q^{2}\right)-\Pi_{b r}\left(q^{2}\right)\right), \\
\Pi_{L L}^{\|}\left(q^{2}\right)=\Pi_{R R}^{\|}\left(q^{2}\right)=\frac{\sin ^{2} \theta}{2 g_{V}^{2}} F^{2}, \quad \Pi_{L R}^{\|}\left(q^{2}\right)=-\frac{\sin ^{2} \theta}{g_{V}^{2}} F^{2} .
\end{gathered}
$$

The relevant quadratic contribution of the gauge bosons to the $4 \mathrm{D}$ partition function is

$$
\begin{aligned}
\mathcal{L}_{\text {eff }} \supset & \left(\frac{q^{\mu} q^{\nu}}{q^{2}}-\eta^{\mu \nu}\right) \frac{1}{4} \Pi_{\text {diag }}\left(q^{2}\right)\left(g^{2} W_{\mu}^{\alpha} W_{\nu}^{\alpha}+g^{\prime 2} B_{\mu} B_{\nu}\right) \\
& +\frac{F^{2} \sin ^{2} \theta}{8 g_{V}^{2}} \frac{q^{\mu} q^{\nu}}{q^{2}}\left(g^{2} W_{\mu}^{\alpha} W_{\nu}^{\alpha}+g^{\prime 2} B_{\mu} B_{\nu}\right) \\
& +\left(\frac{q^{\mu} q^{\nu}}{q^{2}}-\eta^{\mu \nu}\right) \frac{1}{4} \Pi_{L R}\left(q^{2}\right) g g^{\prime} W_{\mu}^{3} B_{\nu}-\frac{q^{\mu} q^{\nu}}{q^{2}} \frac{F^{2} \sin ^{2} \theta}{4 g_{V}^{2}} g g^{\prime} W_{\mu}^{3} B_{\nu} .
\end{aligned}
$$

The mass terms in the effective Lagrangian can be determined from the lowest order in $q^{2}$. Both for the longitudinal and transverse $W$ and $Z$ gauge bosons we get

$$
M_{W}^{2}=\frac{g^{2}}{4} \frac{\sin ^{2} \theta}{g_{V}^{2}} F^{2}, \quad M_{Z}^{2}=\frac{g^{2}+g^{2}}{4} \frac{\sin ^{2} \theta}{g_{V}^{2}} F^{2}
$$

while the photon stays massless. Evidently, the formation of masses proportional to $F^{2}$ follows the EW symmetry breaking scheme and is due to the $S O(5)$ symmetry breaking in the original $H$ field (8) and the misalignment (4).

\section{Left-right correlator and sum rules}

The vacuum polarization amplitudes receive contributions from the new physics (new massive resonances in the loops). To quantify deviations with respect to SM, the EW "oblique" precision parameters were introduced [30,31]. The most relevant for the discussion of the $\mathrm{CH}$ models are the $S$ and $T$ parameters of Peskin and Takeuchi [31]. As we already mentioned, a particular feature of MCHM is that due to the custodial symmetry of the strongly interacting sector, the tree-level correction to the $T$ parameter vanishes. Bearing in mind that the holographic description is meant to be valid only in the large $N_{h c}$ limit, loop corrections are not easily tractable. Thus, we focus on the $S$ parameter connected to the $\Pi_{L R}\left(q^{2}\right)$ as follows:

$$
\begin{aligned}
S & =-4 \pi \Pi_{L R}{ }^{\prime}(0) \\
& =\frac{2 \pi R}{g_{5}^{2}} \frac{\sin ^{2} \theta}{g_{V}^{2}}\left[\gamma_{E}+\psi(1+a)+a \psi_{1}(1+a)\right] .
\end{aligned}
$$

Alternatively, it could be expressed through masses and decay constants:

$$
S=4 \pi \frac{\sin ^{2} \theta}{g_{V}^{2}}\left[\sum_{n} \frac{F_{V}^{2}(n)}{M_{V}^{4}(n)}-\sum_{n} \frac{F_{A}^{2}(n)}{M_{A}^{4}(n)}\right] .
$$

The experimental bounds on the $S$ parameter are essential for the numerical analysis of Sec. VI.

Further, we would like to investigate the validity of the equivalent of the Weinberg sum rules (WSR) that relate the imaginary part of $\Pi_{L R}\left(q^{2}\right)$ to masses and decay constants of vector resonances in the broken and unbroken channels, respectively. We start with the subtracted correlators $\hat{\Pi}_{u n b r}$ and $\hat{\Pi}_{b r}$ of Eqs. (62) and (67), then select a suitable integration circuit and formally obtain

$$
\begin{gathered}
\frac{1}{\pi} \int_{0}^{\infty} \frac{d t}{t} \operatorname{Im} \Pi_{u n b r}(t)=\sum_{n} \frac{F_{V}^{2}(n)}{M_{V}^{2}(n)}, \\
\frac{1}{\pi} \int_{0}^{\infty} \frac{d t}{t} \operatorname{Im} \Pi_{b r}(t)=\sum_{n} \frac{F_{A}^{2}(n)}{M_{A}^{2}(n)}+F^{2} .
\end{gathered}
$$

However, these expressions are ill defined; the external contour does not vanish, and the imaginary part of the poles 
should have been specified. The latter can be done following Vainshtein, i.e., replacing $M_{V}^{2}(n)$ in Eq. (62) with $M_{V}^{2}(n)(1-i \epsilon)$. This prescription reproduces the correct residues. Additionally, the left-hand sides are generically divergent while the sum over resonances possesses an essential singularity on the real axis when the number of resonances $N_{\max }$ encircled in the contour tends to infinity.

We expect to see the convergence properties of the integrals on the left-hand side of (82) and (83) improved when they are gathered in the left-right combination. For the uniformity of notation we introduce the sum $F^{2}=\sum_{n<N_{\max }} F^{2}(n)$ [from Eqn. (68)]. Then,

$$
\begin{aligned}
& \frac{1}{\pi} \int_{0}^{M^{2}\left(N_{\max }\right)} \frac{d t}{t} \operatorname{Im} \Pi_{L R}(t) \\
& \quad=\frac{\sin ^{2} \theta}{g_{V}^{2}} \sum_{n<N_{\max }}\left(\frac{F_{V}^{2}(n)}{M_{V}^{2}(n)}-\frac{F_{A}^{2}(n)}{M_{A}^{2}(n)}-F^{2}(n)\right) .
\end{aligned}
$$

In QCD $\Pi_{L R}$ decays fast enough so that the external contour contribution is negligible when enough resonances are encircled, and this integral vanishes. The equality of Eq. (84) to zero is the first WSR for QCD, and the same arguments allow one to derive the second WSR,

$$
\begin{aligned}
& \frac{1}{\pi} \int_{0}^{M^{2}\left(N_{\max }\right)} d t \operatorname{Im} \Pi_{L R}(t) \\
& \quad=\frac{\sin ^{2} \theta}{g_{V}^{2}} \sum_{n<N_{\max }}\left(F_{V}^{2}(n)-F_{A}^{2}(n)\right)=0 .
\end{aligned}
$$

In fact, it is well known that in QCD, including just the first resonances in the sum provides a fair agreement with phenomenology [32]. In any case, the convergence of the dispersion relation (no subtraction is needed) indicates that the limit $N_{\max } \rightarrow \infty$ could be taken in QCD.

To understand whether the situation is indeed analogous to QCD we should address these two questions: (a) Can the contour integral be neglected? (b) If so, is the integral on the left-hand side converging?

To answer the first question, we consider $\Pi_{L R}\left(Q^{2}\right)$, given explicitly in Eq. (C1) with the Euclidean momenta $Q^{2}=-q^{2}$, and expand it for large $Q^{2}$ (we make use of Stirling's expansion of the $\psi$ function):

$$
\begin{aligned}
\frac{g_{V}^{2} \Pi_{L R}\left(Q^{2}\right)}{Q^{2}}= & \sin ^{2} \theta \frac{2 \kappa^{2} a}{Q^{2}} \frac{R}{g_{5}^{2}}\left(\ln \frac{Q^{2}}{4 \kappa^{2}}+\ln \kappa^{2} \varepsilon^{2}-\frac{2 \kappa^{2} a}{Q^{2}}\right) \\
& +\mathcal{O}\left(\frac{1}{Q^{6}}\right) .
\end{aligned}
$$

This limit is constrained to the (unphysical) region of $\left|\arg Q^{2}\right|<\pi$, while the value on the physical axis $\left(0<\operatorname{Re} q^{2}=-\operatorname{Re} Q^{2}\right)$ stays ill defined (it needs a prescription, such as the one discussed above). However, we are now in the position to discuss the convergence of the outer part of the circuit in Eqs. (84) and (85). Due to the presence of the $\ln Q^{2} / Q^{2}$ and $1 / Q^{2}$ terms, the correlator does not vanish fast enough to make the issue similar to the QCD case. Therefore, the corresponding dispersion relation requires one subtraction constant $c$ to parametrize the part of $\Pi_{L R}\left(Q^{2}\right)$ not determined by its imaginary component,

$$
\frac{\Pi_{L R}\left(Q^{2}\right)}{Q^{2}}=\int_{0}^{\infty} \frac{d t}{t+Q^{2}-i \epsilon} \frac{1}{\pi} \frac{\operatorname{Im} \Pi_{L R}(t)}{t}+c .
$$

In the deep Euclidean region one could use an expansion,

$$
\frac{1}{t+Q^{2}}=\frac{1}{Q^{2}}-\frac{1}{Q^{2}} t \frac{1}{Q^{2}}+\cdots,
$$

and then the dispersion relation in the large $Q^{2}$ limit looks as

$$
\begin{aligned}
\frac{\Pi_{L R}\left(Q^{2}\right)}{Q^{2}}= & c+\frac{1}{Q^{2}} \frac{1}{\pi} \int_{0}^{\infty} \frac{d t}{t} \operatorname{Im} \Pi_{L R}(t) \\
& -\frac{1}{Q^{4}} \frac{1}{\pi} \int_{0}^{\infty} d t \operatorname{Im} \Pi_{L R}(t)+\ldots
\end{aligned}
$$

The next step is to encircle a large, but finite, number of resonances. That is, we take $N_{\max }<\infty$ connected to the UV cutoff via the relation (63). The dispersion relation still holds and Eq. (89) can be compared order-by-order with the large $Q^{2}$ expansion given in Appendix C. Holding to the assumptions made there, we obtain

$$
\int_{0}^{M^{2}\left(N_{\max }\right)} \frac{d t}{t} \operatorname{Im} \Pi_{L R}(t)=0
$$

which establishes the formal validity of the first WSR:

$$
\sum_{n<N_{\max }}\left(\frac{F_{V}^{2}(n)}{M_{V}^{2}(n)}-\frac{F_{A}^{2}(n)}{M_{A}^{2}(n)}-F^{2}(n)\right)=0 .
$$

We further stress that the situation is rather unsimilar to the one of real QCD, essentially because $F^{2}$ is logarithmically dependent on the cutoff. On the other hand, the situation in the holographic $\mathrm{CH}$ scenario is quite analogous to the holographic QCD model of Ref. [21]. We just proved that the sum over vector resonances $\sum_{n<N_{\max }}\left(\frac{F_{V}^{2}(n)}{M_{V}^{2}(n)}-\frac{F_{A}^{2}(n)}{M_{A}^{2}(n)}\right)$ is itself cutoff dependent for $N_{\max } \rightarrow \infty$. This implies that symmetry restoration takes place very slowly in the UV and saturation with the ground state resonance is questionable both in holographic $\mathrm{CH}$ and holographic QCD. It seems fair to conclude that these peculiarities represent a pitfall of holography rather than a characteristic of the $\mathrm{CH}$ model. 
Finally, the nullification of the $\frac{1}{Q^{4}}$ term in (C6) leads to

$$
\frac{1}{\pi} \int_{0}^{M^{2}\left(N_{\max }\right)} d t \operatorname{Im} \Pi_{L R}(t)=0
$$

which formally proves the second WSR of Eq. (85). Again, a cutoff should be imposed to guarantee convergence of both the integral of the imaginary part over the real axis and of the sum over resonances.

\section{HIGHER ORDER CORRELATORS AND COUPLINGS}

Let us write down several 5D interactions of phenomenological interest. At the three-point level they are:

$$
\begin{aligned}
S_{5 \mathrm{D}}^{(3)} \supset & i \frac{R}{g_{5}^{2}} \int d^{5} x e^{-\kappa^{2} z^{2}} z^{-1}\left(\partial_{\mu} A_{\nu}^{A} A^{B \mu} A^{C \nu} \operatorname{Tr} T^{A}\left[T^{B}, T^{C}\right]-\partial_{z} A_{\mu}^{A} A_{z}^{i} A^{B \mu} \operatorname{Tr} T^{A}\left[T^{i}, T^{B}\right]\right. \\
& \left.+\partial_{\mu} A_{z}^{i} A_{z}^{j} A^{A \mu} \operatorname{Tr} T^{i}\left[T^{j}, T^{A}\right]\right)+(f R)^{2} \kappa^{2} \frac{R}{k_{s}} \int d^{5} x e^{-\kappa^{2} z^{2}} z^{-1} \frac{h}{\chi_{\pi}}\left(A_{L}-A_{R}\right)_{\mu}^{\alpha} A_{b r}^{\alpha \mu}
\end{aligned}
$$

To prevent misunderstanding, we specify the left, right, or broken origin of the vector field $A_{\mu}(x, z)$ where it is needed (they go with $\alpha=1,2,3)$. Otherwise, the fields with $i, j=1,2,3,4$ are from the broken sector, and the $A, B, C=1, \ldots, 10$ fields encompass all options. The fourth Goldstone boson field $\pi^{4}(x, z)$ is denoted as $h(x, z)$ henceforth. At the four-point level we have

$$
\begin{aligned}
S_{5 \mathrm{D}}^{(4)} \supset & \frac{R}{4 g_{5}^{2}} \int d^{5} x e^{-\kappa^{2} z^{2}} z^{-1}\left(A_{\mu}^{A} A_{\nu}^{B} A^{C \mu} A^{D \nu} \operatorname{Tr}\left[T^{A}, T^{B}\right]\left[T^{C}, T^{D}\right]-2 A_{z}^{i} A_{\mu}^{A} A_{z}^{j} A^{B \mu} \operatorname{Tr}\left[T^{i}, T^{A}\right]\left[T^{j}, T^{B}\right]\right) \\
& +(f R)^{2} \kappa^{2} \frac{R}{4 k_{s}} \int d^{5} x \frac{e^{-\kappa^{2} z^{2}}}{z} \frac{h^{2}}{\chi_{\pi}^{2}}\left(\left(A_{L \mu}^{\alpha}-A_{R \mu}^{\alpha}\right)^{2}-2 A_{b r \mu}^{\alpha 2}\right) .
\end{aligned}
$$

The commutators there can be simplified with the Lie algebra of $S O(5)$,

$$
\begin{aligned}
{\left[T_{L}^{\alpha}, T_{L}^{\beta}\right]=i \varepsilon^{\alpha \beta \delta} T_{L}^{\delta}, } & {\left[T_{R}^{\alpha}, T_{R}^{\beta}\right]=i \varepsilon^{\alpha \beta \delta} T_{R}^{\delta}, \quad\left[T_{L}^{\alpha}, T_{R}^{\beta}\right]=0, \quad \alpha, \beta, \delta=1,2,3, } \\
{\left[T^{a}, \hat{T}^{i}\right]=\hat{T}^{j}\left(t^{a}\right)^{j i}, } & {\left[\hat{T}^{i}, \hat{T}^{j}\right]=\left(t_{a}\right)^{j i} T^{a}, \quad a=1, \ldots, 6, \quad i=1, \ldots, 4 . }
\end{aligned}
$$

Here $t^{a}=\left\{t_{L}^{\alpha}, t_{R}^{\alpha}\right\}$ [see the definition after Eq. (2)].

The expressions for $S_{5 \mathrm{D}}^{(3)}$ and $S_{5 \mathrm{D}}^{(4)}$ are already simplified with the gauge choice $A_{z}^{a}=0$ in the unbroken channel. The Higgs-related terms proportional to the $S O(5)$ breaking $(f R)^{2}$ come from the square of the covariant derivative in Eq. (7). Taking into account that in the broken sector imposed with $\xi=1$ we also had the gauge-Higgs bosons from $A_{z}^{i}=\frac{\partial_{z} \pi^{i}}{\chi_{\pi}}$, we reveal the following interactions involving the Higgs boson from the $F_{M N}^{2}$ term:

$$
\frac{R}{2 g_{5}^{2}} \int d^{5} x \frac{e^{-\kappa^{2} z^{2}}}{z}\left[\frac{\partial_{z} h}{\chi_{\pi}}\left(A_{L}-A_{R}\right)_{\mu}^{\alpha} \partial_{z} A_{b r}^{\alpha \mu}+\frac{1}{4}\left(\frac{\partial_{z} h}{\chi_{\pi}}\right)^{2}\left(\left(A_{L \mu}^{\alpha}-A_{R \mu}^{\alpha}\right)^{2}+A_{b r \mu}^{\alpha 2}\right)\right]
$$

We are interested in triple and quartic couplings between the Higgs boson and the SM gauge bosons. In the standard MCHM picture these interactions have a given parametrization in the coordinate space

$$
\begin{gathered}
g_{h W W}^{\mathrm{SM}} \cos \theta W_{\mu}^{+} W^{-\mu} h+g_{h Z Z}^{\mathrm{SM}} \cos \theta \frac{1}{2} Z_{\mu} Z^{\mu} h+\frac{\cos 2 \theta}{4}\left(g^{2} W_{\mu}^{+} W^{-\mu}+\frac{g^{2}+g^{\prime 2}}{2} Z_{\mu} Z^{\mu}\right) h h, \\
g_{h W W}^{\mathrm{SM}}=g M_{W}, \quad g_{h Z Z}^{\mathrm{SM}}=\sqrt{g^{2}+g^{\prime 2}} M_{Z},
\end{gathered}
$$

with $W_{\mu}^{ \pm}=\frac{W_{\mu}^{1} \mp i W_{\mu}^{2}}{\sqrt{2}}, \quad Z_{\mu}=\frac{1}{\sqrt{g^{2}+g^{2}}}\left(g W_{\mu}^{3}-g^{\prime} B_{\mu}\right)$. 
In our 5D model, the effective couplings for $h W W$ and $h h W W$ originate from

$$
\begin{aligned}
\mathcal{L}_{\text {eff }} \supset & i \frac{g^{2}}{4 g_{V}^{2}} h(q) W^{\alpha \mu}\left(k_{1}\right) W^{\beta \nu}\left(k_{2}\right)\left\langle h(q)\left|\tilde{\mathcal{O}}_{L \mu}^{\alpha}\left(k_{1}\right) \tilde{\mathcal{O}}_{L \nu}^{\beta}\left(k_{2}\right)\right| 0\right\rangle \\
& +i \frac{g^{2}}{4 g_{V}^{2}} h\left(q_{1}\right) h\left(q_{2}\right) W^{\alpha \mu}\left(k_{1}\right) W^{\beta \nu}\left(k_{2}\right)\left\langle h\left(q_{1}\right) h\left(q_{2}\right)\left|\tilde{\mathcal{O}}_{L \mu}^{\alpha}\left(k_{1}\right) \tilde{\mathcal{O}}_{L \nu}^{\beta}\left(k_{2}\right)\right| 0\right\rangle .
\end{aligned}
$$

$Z$ boson couplings can be taken into consideration after addition of the terms generated by $\tilde{\mathcal{O}}_{L \mu}^{3} \tilde{\mathcal{O}}_{R \nu}^{3}, \tilde{\mathcal{O}}_{R \mu}^{3} \tilde{\mathcal{O}}_{L \nu}^{3}$, and $\tilde{\mathcal{O}}_{R \mu}^{3} \tilde{\mathcal{O}}_{R \nu}^{3}$ operator combinations. Their derivation follows closely that of the $W^{+} W^{-}$, so we just include them in the final result.

Particularities of calculating the matrix elements in (98) and (99) can be found in Appendix D. The couplings to the EW gauge bosons appear in the effective Lagrangian as

$$
\begin{aligned}
\mathcal{L}_{\text {eff }} \supset & \frac{g_{h W W}^{\mathrm{SM}} \cos \theta}{\sqrt{2} g_{V}} \cdot \frac{1}{2}\left(W^{+\mu}\left(k_{2}\right) W_{\mu}^{-}\left(k_{1}\right)+W^{-\mu}\left(k_{2}\right) W_{\mu}^{+}\left(k_{1}\right)\right) h(q) \\
& +\frac{g^{2} \cos 2 \theta}{8 g_{V}^{2}} \cdot \frac{1}{2}\left(W_{\mu}^{+}\left(k_{1}\right) W^{-\mu}\left(k_{2}\right)+W_{\mu}^{-}\left(k_{1}\right) W^{+\mu}\left(k_{2}\right)\right) h\left(q_{1}\right) h\left(q_{2}\right) \\
& +\frac{g_{h Z Z}^{\mathrm{SM}} \cos \theta}{\sqrt{2} g_{V}} \cdot \frac{1}{2} Z^{\mu}\left(k_{2}\right) Z_{\mu}\left(k_{1}\right) h(q) \\
& +\frac{\left(g^{2}+g^{\prime 2}\right) \cos 2 \theta}{8 g_{V}^{2}} \cdot \frac{1}{2} Z_{\mu}\left(k_{1}\right) Z^{\mu}\left(k_{2}\right) h\left(q_{1}\right) h\left(q_{2}\right), \\
& g_{h W W}^{\mathrm{SM}}=\frac{g^{2} F \sin \theta}{2 g_{V}}, \quad g_{h Z Z}^{\mathrm{SM}}=\frac{\left(g^{2}+g^{\prime 2}\right) F \sin \theta}{2 g_{V}},
\end{aligned}
$$

where the factors in the last line indeed correspond to the SM notation of Eq. (97) due to the definition of masses in Eq. (79). The only thing missing to have the exact MCHM factors of Eq. (96) is the proper choice of the so-far free parameter

$$
g_{V}=\frac{1}{\sqrt{2}}
$$

Note that this value is obtained in the approximation $M_{W}^{2} \ll 4 \kappa^{2}$ assumed in the calculations of Appendix D.

Let us now turn to the part of Eq. (93) independent of $A_{z}$ and the Higgs modes

$$
i \frac{R}{g_{5}^{2}} \int d^{5} x e^{-\kappa^{2} z^{2}} z^{-1} \partial_{\mu} A_{\nu}^{A} A^{B \mu} A^{C \nu} \operatorname{Tr} T^{A}\left[T^{B}, T^{C}\right]
$$

The trace is proportional to the epsilon-tensor if none of the three fields is $A_{b r}^{4}$; in the opposite case we obtain a Kronecker delta.
There is an interaction between the three vector 5D fields in Eq. (106). In order to procure a coupling of a vector resonance to two EW gauge bosons one of the fields should be taken in its KK representation, while the other two should be given by their bulk-to-boundary propagators coupled later to the corresponding gauge field sources. The details of these calculations are presented in Appendix E. Here, we limit ourselves to listing just the interactions between the EW bosons and the ground states of the composite resonances

$$
\begin{aligned}
\mathcal{L}_{\text {eff }} \supset & \frac{1}{2} W_{\mu_{2}}^{\alpha}\left(q_{2}\right) W_{\mu_{3}}^{\beta}\left(q_{3}\right) \operatorname{Lor}^{\mu_{1} \mu_{2} \mu_{3}}\left(q_{1}, q_{2}, q_{3}\right)\left(-i \varepsilon^{\alpha \beta \delta}\right) \\
& \times\left(A_{\mu_{1}}^{L \delta}\left(q_{1}\right) g_{L W W}+A_{\mu_{1}}^{R \delta}\left(q_{1}\right) g_{R W W}\right. \\
& \left.-A_{\mu_{1}}^{B r \delta}\left(q_{1}\right) g_{B r W W}\right) \\
+ & W_{\mu_{2}}^{\alpha}\left(q_{2}\right) B_{\mu_{3}}\left(q_{3}\right) \operatorname{Lor}^{\mu_{1} \mu_{2} \mu_{3}}\left(q_{1}, q_{2}, q_{3}\right)\left(-i \varepsilon^{\alpha 3 \delta}\right) \\
& \times\left(A_{\mu_{1}}^{L \delta}\left(q_{1}\right) g_{L W B}+A_{\mu_{1}}^{R \delta}\left(q_{1}\right) g_{R W B}\right)
\end{aligned}
$$


where the notation $\operatorname{Lor}^{\mu_{1} \mu_{2} \mu_{3}}\left(q_{1}, q_{2}, q_{3}\right)$ was given in Appendix E, and we introduced

$g_{L / R W W}=\frac{g^{2}}{4 g_{V}^{2}} \sqrt{\frac{R}{2 g_{5}^{2}}}\left[1 \pm \cos \theta+a \sin ^{2} \theta\left(a \psi_{1}(1+a)-1\right)\right]$,

$$
\begin{gathered}
g_{B r W W}=\frac{g^{2}}{4 g_{V}^{2}} \sqrt{\frac{R}{g_{5}^{2}}} \frac{\sin \theta}{1+a}, \\
g_{L W B}=g_{R W B}=\frac{g g^{\prime}}{4 g_{V}^{2}} \sqrt{\frac{R}{2 g_{5}^{2}}} a \sin ^{2} \theta\left[1-a \psi_{1}(1+a)\right] .
\end{gathered}
$$

The numerical values of these couplings will be estimated in the next section.

\section{NUMERICAL RESULTS FOR MASSES AND COUPLINGS}

A very stringent limit on any new physics contribution comes from the experimental bounds on the $S$ parameter, calculated using 5D techniques in Eq. (80) or (81). Recent EW precision data (see Ref. [33]) constrains it to the region

$$
S=-0.01 \pm 0.10
$$

There are four model parameters in our expression for $S$. The parameter $g_{V}$ is assumed to be fixed as in Eq. (105), while the other three $\left(\sin \theta, a\right.$, and $\left.\frac{R}{g_{5}^{2}}\right)$ at this moment remain unconstrained by the theoretical construction but for their inherent positivity. We recall that $a$ is related to the $S O(5)$ symmetry breaking parametrized in $H$ as $f(z)$ [see Eq. (8)]; at $a=0$ there is no breaking, the unbroken and broken vector modes have the same mass. $\frac{R}{g_{5}^{2}}$ could be evaluated, in principle, by comparing holographic two-point function to the perturbative calculation of the Feynman diagram (e.g., of a hyperfermion loop) at the leading order in large $Q^{2}$ momenta, as it is usually done in the holographic realizations of QCD. As we would expect to get the hypercolor trace in the loop, it could be estimated that there is a proportionality $\frac{R}{g_{5}^{2}} \propto N_{h c}^{p}$ (power $p$ depends on the particular representation). However, we deliberately made no hypothesis on the fundamental substructure, and could only expect that very large values of $\frac{R}{g_{5}^{2}}$ correspond to the large- $N_{h c}$ limit. To have an idea of the scale of this quantity, we recall that for $N_{c}=3$, QCD one has $\frac{R}{g_{5}^{2}} \sim 0.3$ [21].

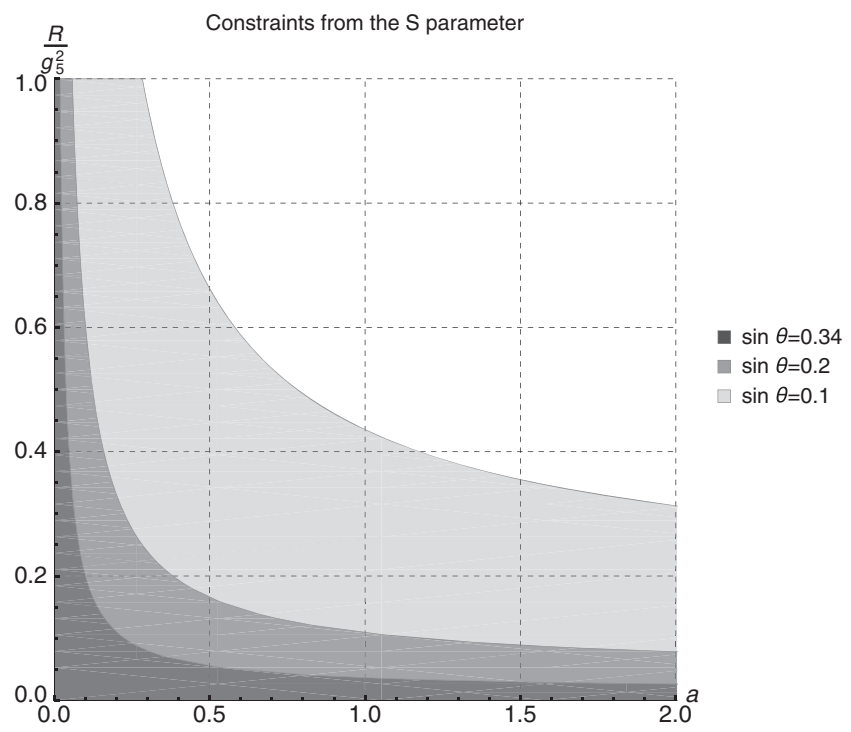

FIG. 2. The $\left(\sin \theta, a, R / g_{5}^{2}\right)$ parameter region allowed by the $S$ parameter restraints.

We present the effect of the current $S$-constraint on the $\left(\sin \theta, a, R / g_{5}^{2}\right)$ plane in Fig. 2. The larger the value of $\sin \theta$, the smaller the allowed region for $a$ and $R / g_{5}^{2}$. We only consider $\sin \theta \leq 0.34$ due to the present bounds on the misalignment in MCHM [34] [for the SM fermions in the spinoral representation of $S O(5)]$. That bound is valid under the assumption that the coupling of the Higgs boson to gauge bosons is $\kappa_{V}=\sqrt{1-\sin ^{2} \theta}$, and it was demonstrated in Sec. V that this is the case of our holographic model, too. Otherwise, we can take a more model independent estimation from the latest ATLAS and CMS combined measurements with the LHC Run 1 dataset [33] that leads to $\kappa_{V}=1.04 \pm 0.05$ at one standard deviation. Taken at two standard deviations, it results once again in $\sin \theta \leq 0.34$. Nevertheless, stricter (lower) bounds could also be encountered in the literature (Run 2 analyses [35,36], for instance).

No information on the mass scale $\kappa$ could be retrieved from the EW precision data. However, we can relate it to the low-energy observables through the definition of the $W$ boson mass in Eq. (79). It is connected to the EWSB scale $v=246 \mathrm{GeV}$ and we can equate

$$
M_{W}^{2}=\frac{g^{2} v^{2}}{4}=\frac{g^{2} F^{2} \sin ^{2} \theta}{4 g_{V}^{2}} .
$$

With $F$ given in Eq. (50), the following condition on $\kappa$ is valid: 
TABLE I. Different predictions of the minimal vector masses for $\sin \theta=0.1,0.2$, and 0.34 .

\begin{tabular}{cccccc}
\hline \hline $\sin \theta$ & $\frac{R}{g_{5}^{2}}$ & $a$ & $M_{*}=M_{V}(0), \mathrm{TeV}$ & $M_{A}(0), \mathrm{TeV}$ & $\sim N_{\max }$ \\
\hline 0.1 & 0.1 & 266.3 & 0.22 & 3.68 & $>20 \mathrm{k}$ \\
0.1 & 0.3 & 2.212 & 1.28 & 2.29 & 740 \\
0.1 & 1 & 0.283 & 1.88 & 2.13 & 340 \\
0.1 & 10 & 0.022 & 2.10 & 2.12 & 270 \\
0.2 & 0.1 & 1.176 & 1.79 & 2.64 & 93 \\
0.2 & 0.3 & 0.225 & 2.28 & 2.52 & 58 \\
0.2 & 1 & 0.058 & 2.43 & 2.50 & 50 \\
0.2 & 10 & 0.006 & 2.49 & 2.50 & 48 \\
0.34 & 0.1 & 0.225 & 2.84 & 3.14 & 12 \\
0.34 & 0.3 & 0.065 & 3.00 & 3.09 & 11 \\
0.34 & 1 & 0.019 & 3.05 & 3.08 & 10 \\
0.34 & 10 & 0.002 & 3.07 & 3.08 & 10 \\
\hline \hline
\end{tabular}

$\frac{g_{V}^{2} v^{2}}{\sin ^{2} \theta}+\frac{2 \kappa^{2} R}{g_{5}^{2}} a\left(\ln \kappa^{2} \varepsilon^{2}+2 \gamma_{E}+\psi(1+a)\right)=0$.

Let us further set

$$
\varepsilon=\frac{1}{\Lambda_{\text {cut-off }}} \simeq \frac{1}{4 \pi f_{C H}}=\frac{\sin \theta}{4 \pi v}
$$

Here $\Lambda_{\text {cut-off }}=\Lambda_{\mathrm{CH}} \simeq 4 \pi f_{C H}$ is the range of validity of the effective theory of the composite resonances, which could be postulated as a natural cutoff in the present bottom-up model. We can also rework the connection between the number of resonances cutoffs $N_{\max }$ and $\varepsilon$ :

$$
N_{\max }=16 \pi^{2} \frac{v^{2}}{\kappa^{2} \sin ^{2} \theta} e^{-2 \gamma_{E}}
$$

Setting $g_{V}=\frac{1}{\sqrt{2}}$, we collect the results in Table I. There, we substitute the estimation of $\kappa$ with that of the characteristic mass $M_{*}=\sqrt{4 \kappa^{2}}$, equal to the mass of the ground vector state- the lightest massive state in our spectrum. We take the values of $a$ saturating the $S$-bound, thus, these are the minimal estimations for $M_{*}$. Should it be found that $S$ is $p$ times smaller, our evaluations for $M_{*}$ become roughly $p$ times larger. For a given set of $\frac{R}{g_{5}^{2}}$ and $\sin \theta$ lower values of $a$ are permitted and result in larger $M_{*}$. In addition, larger $a$ leads to larger splitting between vector fields aligned in different (unbroken and broken) directions. It is evident from Table I that the splitting almost disappears starting from $\frac{R}{g_{5}^{2}}=10$ for the demonstrated values of $\sin \theta$. We also notice that the effective " $N_{h c}$-infinity" is heralded by the degenerate vector masses in the unbroken and broken sectors and starts rather early because the $\frac{R}{g_{5}^{2}}=10$ fit brings similar results to, say, $\frac{R}{g_{5}^{2}}=1000$. It is an interesting observation, because in the original AdS/CFT conjecture the strongly coupled Yang-Mills theory on the 4D side of the correspondence should be in the limit $N_{c} \gg 1$. Of course, in phenomenological AdS/QCD models the duality is commonly extended for the finite values of $N_{c}$, so we take into consideration a set of smaller $\frac{R}{g_{5}^{2}}$ as well.

In Fig. 3 we depict a broader range of $M_{*}$ values. The dependencies on the model parameters could be easily traced from there. In the parameter space $\left(\sin \theta, a, \frac{R}{g_{5}^{2}}\right)$ we can fix any two values, then the growth of the third parameter results in lower $M_{*}$ (as long as it does not appear in the prohibited zone). Pursuing a higher degree of breaking $a$ results in unlikely small masses in the areas that are not well-restrained by the $S$ parameter. We speak of masses below $2 \mathrm{TeV}$ at smaller values of $\frac{R}{g_{5}^{2}}$ and $\sin \theta$ (a concrete reference could be seen in the first line of Table I). Higher values of other two parameters are more efficiently cut off by the $S$ bound. In general, $2.0-4.0 \mathrm{TeV}$ states are expected. We also recollect that in a tower of resonances of one type we have a square root growth with the number of a resonance. Thus, for rather low value of $M_{*}$ there is a tower with several comparatively low-lying states. For instance, for the input set $\left(\sin \theta, a, R / g_{5}^{2}\right)=(0.1,2.2,0.3)$ we have $M_{*}=1.3 \mathrm{TeV}$ and the tower masses are $M_{V}(n)=\{1.3,1.8,2.3,2.6, \ldots\} \mathrm{TeV}$.

In Fig. 4 we present the numerical analysis resulting from Eqs. (110), (111), and (112), showing the possible values of the couplings between the left, right, and broken resonances and a $W^{+} W^{-}$or $W^{3} B$-pair. It is clear that the left resonances couple more strongly than the right ones thanks to the dampening the latter get with $\cos \theta$ being rather close to 1 . All the $W W$ couplings exhibit a logarithmic growth with $\frac{R}{g_{5}^{2}}$. The parameter $a$ was taken to be saturating the $S$-bound of Fig. 2 and is rendered quite close to zero at higher values of $R / g_{5}^{2}$ especially for larger $\sin \theta$. The coupling including the $B$ gauge boson is rather small in comparison to the $W W$ ones due to the direct proportionality to $a$, and it vanishes exactly for $a=0$.

In order to show the impact of $a$ on $W W$ couplings in more detail we provide the same computation in Fig. 5, imposing $a=0$ by hand for the fit with $\sin \theta=0.1$ (the most illustrative case). The saturation now is reached sooner than in the top panel of Fig. 4, but at the major part of the $R / g_{5}^{2}$ axis the scale of $S O(5)$ breaking is of little consequence for the couplings discussed. This only becomes more prominent at larger values of $\sin \theta$. It is also noticeable, however, that in the region $R / g_{5}^{2} \lesssim 0.5$, where the effect of the $S$ constraint on $a$ is largely decreased, the change is rather substantial. At the same 

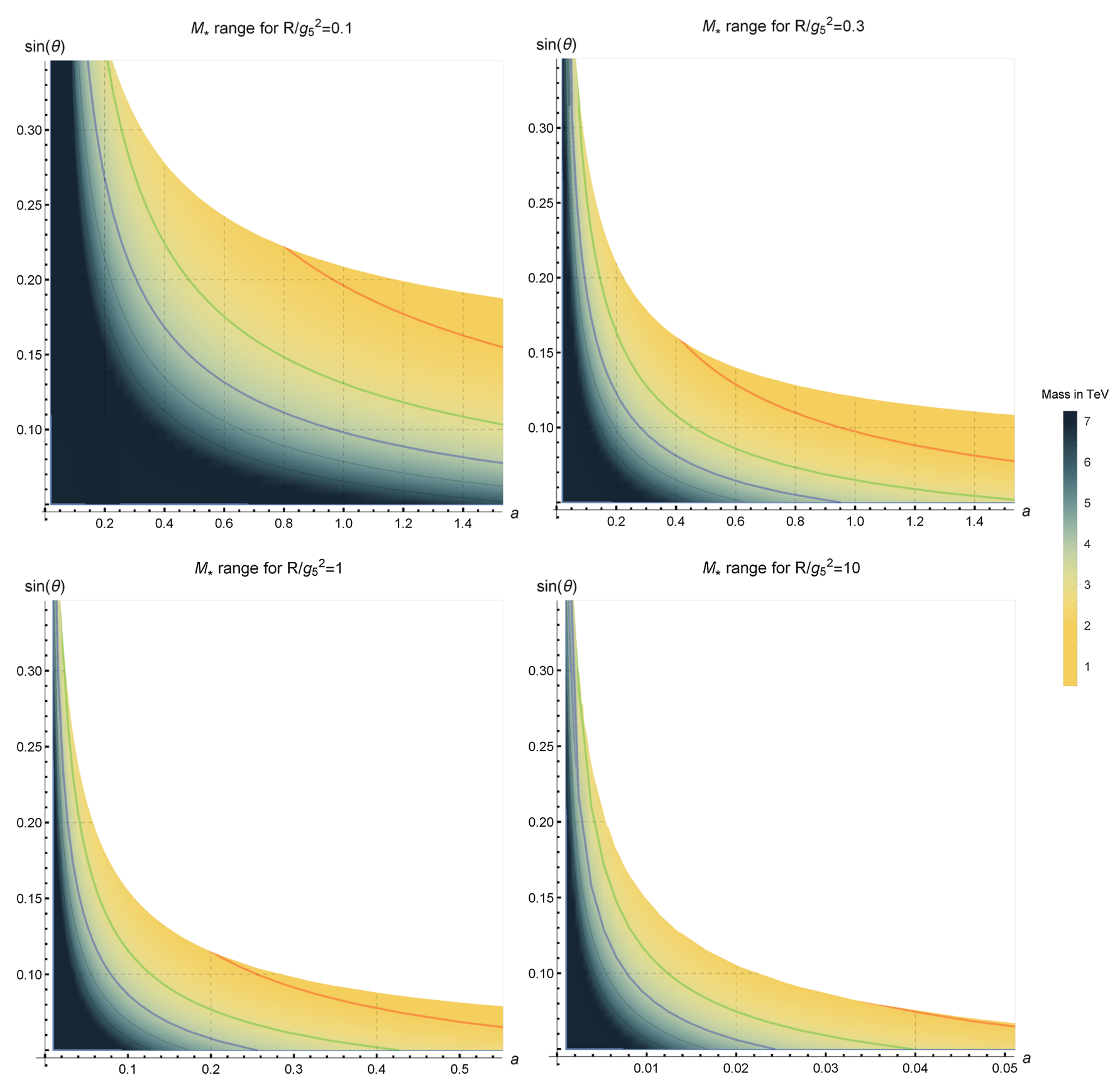

FIG. 3. The density plots of $M_{*}$ for different values of $R / g_{5}^{2}$. The colored curves represent the lines of constant $M_{*}$ : the red one, $M_{*}=2 \mathrm{TeV}$; the green one, $M_{*}=3 \mathrm{TeV}$; the blue one, $M_{*}=4 \mathrm{TeV}$; and successive black curves for higher integer values. The white area represents the sector prohibited by the $S$ bound.

time, this area turns out relevant if we assume that the $\mathrm{CH}$ value is close to the QCD one, or if we take into account the estimations of these couplings made in other studies.

It is not easy to make a comparison of the values of the couplings obtained here with the possible experimental bounds on them because in the analyses of the LHC experimental data on resonances decaying into $W W$ or
$W Z$ pairs some benchmark signal models are normally used (Kaluza-Klein graviton in extra dimension, extended gauge model of $W^{\prime}$ and $Z^{\prime}$, and others). However, in the more model independent framework of Ref. [37] we find that the characteristic scale for the couplings is of order $0.001 \div 0.010$. $g_{B r W W}$ and especially $g_{L W W}$ tend to be much larger unless computed at very small $R / g_{5}^{2}$ (we would put a 


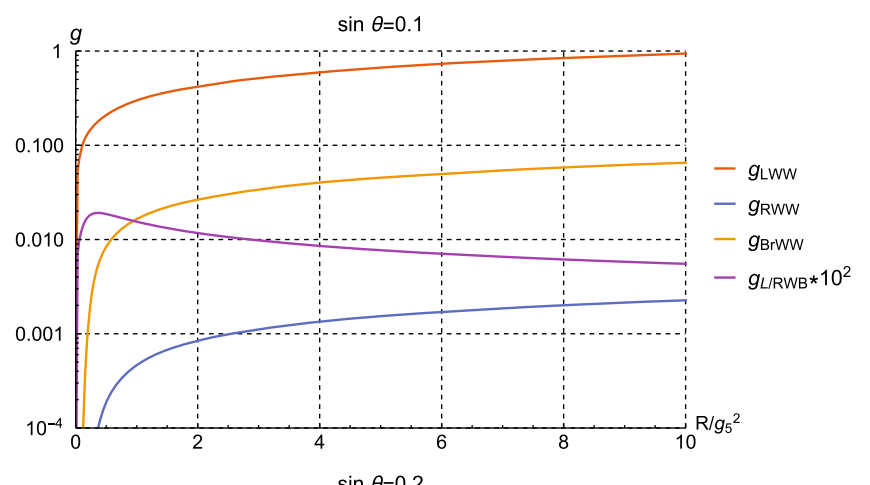

\section{CONCLUSIONS}

In this study we used the bottom-up holographic approach to have a fresh look at nonperturbative aspects of $\mathrm{CH}$ models with a global breaking pattern $S O(5) \rightarrow$ $S O(4)$ and a gauge group misaligned with the unbroken group. With the purpose of being as close as possible to the characteristics of a confining theory (presumed to be underlying the EWSBS) we chose to work in a 5D SW framework inspired by effective models of QCD and consisting of a generalized sigma model coupled to both the composite resonances and the SM gauge bosons. The 5D model is similar to that of the successful AdS/QCD constructions, specifically to our earlier work [21], and depends on the two Ansätze functions: the SW dilaton profile $\Phi(z)$ and the symmetry-breaking $f(z)$. The microscopic nature of the breaking, besides being triggered by some new strong interactions with a hypercolor group, is factored out and every effort has been taken to make predictions as independent of it as possible.

We investigated the dynamics of ten vector (unbroken and broken) and four Goldstone boson (one of them related to the Higgs boson) 5D fields. Though for the unbroken vectors the situation is rather similar to a generic AdS/QCD model, in the broken sector we have additionally adopted a gauge that relates the Goldstone fields to the fifth component of the bulk vector field $A_{z}^{i}$. That is not just a gauge-Higgs construction because there are also definite independent Goldstone modes in the bulk scalar field $H$, the structure of which provides the $S O(5)$ breaking. The resulting Goldstone description is quite different from that of the vector fields, for instance, their UV boundary conditions are set following the gauge-Higgs implementation. In the end, the proposed procedure is ratified by the agreement of the $h W W$ and $h h W W$ characteristic couplings to those of the general MCHM. The Higgs boson remains massless as long as we do not take into account the quantum corrections.

In the paper we lay emphasis on the following issues of phenomenological interest:

(i) derivation of the spectra of the new states in the broken and unbroken channels;

(ii) connection to the EW sector (masses of the gauge bosons and the electroweak precision observables);

(iii) triple couplings of the new heavy resonances to $W^{+} W^{-}$and $W^{ \pm} B$

(iv) in-depth analysis of the realization of the first and second Weinberg sum rules and the study of their convergence.

The holographic effective theory describes the composite resonances; their maximum number $N_{\max }$ is found to be related to the theory natural UV cutoff $\varepsilon$. Adhering to one of these cutoffs is necessary to derive relations involving resonance decay constants and masses. The latter stay cutoff independent as befits physical observables. The only, but very significant exception, is the "pion" decay constant

FIG. 5. Example of the couplings estimated for a completely vanishing value of $a$. 
$F$. We made a hypothesis that $\varepsilon$ can be taken as being related to the characteristic range of the $\mathrm{CH}$ effective theory, and provided numerical estimations for the value of $N_{\max }$. Moreover, the two Weinberg sum rules hold their validity just in a formal sense as the sum over resonances has to be cut off. The sum rules are logarithmically divergent, and this implies that they are not saturated at all by just the first resonance. We believe it to be a common feature of AdS/CFT models, detached from the particularities of our setup, as it is also present in holographic QCD. We can regard it as a general serious flaw of the bottom-up holographic models, and hence a realistic $\mathrm{CH}$ theory could also have the sum rules more similar to those of actual QCD.

The minimal set of input parameters in our model is: $\sin \theta$, $a$, and $\frac{g_{5}^{2}}{R}$. There are constraints coming from the $W$ mass (EW scale), the $S$ parameter and the existing experimental bounds on $\kappa_{V}(\sin \theta)$. Their consideration allows us to estimate the masses for the composite resonances. It is not difficult to find areas in the parameter space where a resonance between 2 and $3 \mathrm{TeV}$ is easily accommodated. The presented technique offers the possibility of deriving trilinear couplings of a type $W W, W B-$ new composite resonance. They are of interest because the SM gauge boson scattering is regarded as the process for the new vector resonance production in collider experiments.

It is compelling to extend the proposed framework to other nonminimal symmetry breaking patterns, especially the ones that could be supported by a nonexotic theory at the microscopic level. Then, it would be reasonable to include more quantities of physical interest into the analysis. Also, it would be more interesting to develop the connection to the SM fermions. Despite the fact that the idea behind our framework prohibits them from entering into the bulk, their mixing with the new fundamental constituents of the strongly interacting sector is possible and could be explored in more detail.

\section{ACKNOWLEDGMENTS}

We acknowledge financial support from the following Grants No. FPA2016-76005-C2-1-P and No. PID2019105614 GB-C21 (MICINN), and No. 2017SGR0929
(Generalitat de Catalunya). A. K. acknowledges the financial support of the fellowship BES-2015-072477. The activities of I.C.C.U.B. are supported from the State Agency for Research of the Spanish Ministry of Science and Innovation through the Unit of Excellence Maria de Maeztu 2020-2023 Award No. (CEX2019-000918-M).

\section{APPENDIX A: CONFLUENT HYPERGEOMETRIC EQUATION AND ITS SOLUTIONS}

The confluent hypergeometric equation is given as

$$
y \varphi^{\prime \prime}(y)+(c-y) \varphi^{\prime}(y)-a \varphi(y)=0 .
$$

The values of parameters $a$ and $c$ define the types of solution one would get [38]. We abstain from considering solutions whose IR asymptotics tend to explode.

For the positive integer values $c=1,2,3, \ldots$ we have

$$
\varphi(y)=C_{11} F_{1}(a, c ; y)+C_{2} \Psi(a, c ; y),
$$

where ${ }_{1} F_{1}(a, c ; y)$ is called the Kummer function and $\Psi(a, c ; y)$ is the Tricomi function.

However, in the paper we frequently meet the cases of nonpositive integer $c .{ }_{1} F_{1}(a, c ; y)$ has poles at $c=0,-1,-2, \ldots$, while the Tricomi function can generally be analytically continued to any integer value of $c$. In that situation we can choose another two solutions from the fundamental system of solutions:

$$
\varphi(y)=C_{1} y^{1-c}{ }_{1} F_{1}(a-c+1,2-c ; y)+C_{2} \Psi(a, c ; y) .
$$

Let us discuss several properties of these confluent hypergeometric functions [38]:

(i) The Tricomi functions with different arguments are related via

$$
\Psi(a, c ; y)=y^{1-c} \Psi(a-c+1,2-c ; y) .
$$

(ii) The Tricomi function exhibits a logarithmic behavior for all integer values of $c$. Specifically, for the case $c=1-n, n=0,1,2, \ldots$, one has

$$
\begin{aligned}
\Psi(a, 1-n ; y)= & \frac{(n-1) !}{\Gamma(a+n)} \sum_{r=0}^{n-1} \frac{(a)_{r} y^{r}}{(1-n)_{r} r !}+\frac{(-1)^{n-1}}{n ! \Gamma(a)}\left({ }_{1} F_{1}(a+n, n+1 ; x) y^{n} \ln y\right. \\
& \left.+\sum_{r=0}^{\infty} \frac{(a+n)_{r}}{(n+1)_{r}}[\psi(a+n+r)-\psi(1+r)-\psi(1+n+r)] \frac{y^{n+r}}{r !}\right),
\end{aligned}
$$

here the Pochhammer symbol is $(a)_{n}=1 \cdot a \cdot(a+1) \ldots(a+n-1)=\Gamma(a+n) / \Gamma(a), \psi(a)$ is the digamma function, and the first sum is absent for the case $n=0$. 
(iii) The Tricomi function has an infinite sum representation involving the generalized Laguerre polynomials

$$
\Gamma(a) \Psi(a, 1+m ; y)=\sum_{n=0}^{\infty} \frac{L_{n}^{m}(y)}{n+a} .
$$

(iv) The Kummer function is a (finite) series solution, ${ }_{1} F_{1}(a, c ; y)=\sum_{n=0}^{\infty} \frac{(a)_{n}}{(c)_{n}} \frac{y^{n}}{n !}$, that has a natural connection with the generalized Laguerre polynomials (for integer $n>0, m>0$ )

$$
L_{n}^{m}(y)=\frac{(m+1)_{n}}{n !}{ }_{1} F_{1}(-n, m+1, y) .
$$

\section{APPENDIX B: DERIVATION OF THE EOM IN THE BROKEN SECTOR WITH $\xi=1$}

Let us assume $A_{z}^{i}=\frac{\partial_{z} \pi^{i}}{\chi_{\pi}}$ directly in Eqs. (31)-(33). Then, the system on $A_{\mu}^{i}$ and $\pi^{i}$ simplifies to

$$
\begin{gathered}
\partial_{z} \frac{e^{-\Phi(z)}}{z} \partial_{z} A_{\mu}^{i}-\frac{e^{-\Phi(z)}}{z} \square A_{\mu}^{i}-\frac{2 g_{5}^{2} f^{2}(z) R^{2}}{k_{s}} \frac{e^{-\Phi(z)}}{z^{3}} A_{\mu}^{i} \\
-\partial_{\mu}\left(\partial_{z} \frac{e^{-\Phi(z)}}{z} \partial_{z} \frac{\pi^{i}}{\chi_{\pi}}-\frac{2 g_{5}^{2} f^{2}(z) R^{2}}{k_{s}} \frac{e^{-\Phi(z)}}{z^{3}} \frac{\pi^{i}}{\chi_{\pi}}\right)=0, \\
\partial^{\mu} A_{\mu}^{i}=\square \frac{\pi^{i}}{\chi_{\pi}} .
\end{gathered}
$$

The condition of Eq. (34) holds, and together with Eq. (B2) it implies that

$$
\square^{2} \frac{\pi^{i}}{\chi_{\pi}}=0
$$

With the use of the identity $A_{\mu}^{i \|}=\frac{\partial_{\mu} \partial^{\nu}}{\square} A_{\nu}^{i}=\partial_{\mu} \frac{\pi^{i}}{\chi_{\pi}}$, the longitudinal part in Eq. (B1) transforms into

$$
\begin{aligned}
& \partial_{z} \frac{e^{-\Phi(z)}}{z} \partial_{z} A_{\mu}^{i \|}-\frac{2 g_{5}^{2} f^{2}(z) R^{2}}{k_{s}} \frac{e^{-\Phi(z)}}{z^{3}} A_{\mu}^{i \|} \\
& -\partial_{\mu}\left(\partial_{z} \frac{e^{-\Phi(z)}}{z} \partial_{z} \frac{\pi^{i}}{\chi_{\pi}}+\frac{e^{-\Phi(z)}}{z} \square \frac{\pi^{i}}{\chi_{\pi}}-\frac{2 g_{5}^{2} f^{2}(z) R^{2}}{k_{s}} \frac{e^{-\Phi(z)}}{z^{3}} \frac{\pi^{i}}{\chi_{\pi}}\right) \\
& =0 .
\end{aligned}
$$

All things considered, one of the possible solutions is this set of simultaneously fulfilled equations:

$$
\partial_{z} \frac{e^{-\Phi(z)}}{z} \partial_{z} A_{\mu}^{i \|}-\frac{2 g_{5}^{2} f^{2}(z) R^{2}}{k_{s}} \frac{e^{-\Phi(z)}}{z^{3}} A_{\mu}^{i \|}=0,
$$

$$
\partial_{z} \frac{e^{-\Phi(z)}}{z} \partial_{z} \frac{\pi^{i}}{\chi_{\pi}}-\frac{2 g_{5}^{2} f^{2}(z) R^{2}}{k_{s}} \frac{e^{-\Phi(z)}}{z^{3}} \frac{\pi^{i}}{\chi_{\pi}}=0
$$

$$
\square \frac{\pi^{i}}{\chi_{\pi}}=0
$$

while the transverse mode keeps being described by Eq. (38).

With this exercise we intend to be reassured that the masslessness of the Goldstones agrees with EOMs (37), (38), and (39) given in the main body of the paper.

\section{APPENDIX C: LARGE $Q^{2}$ EXPANSION OF THE CORRELATOR $\Pi_{L R}$}

Here we perform the large $Q^{2}$ expansion of $\Pi_{L R}$ given by

$$
\begin{aligned}
g_{V}^{2} \Pi_{L R}\left(Q^{2}\right) \\
=\frac{R}{2 g_{5}^{2}} Q^{2} \sin ^{2} \theta\left\{\psi\left(1+\frac{Q^{2}}{4 \kappa^{2}}\right)-\psi\left(1+\frac{Q^{2}}{4 \kappa^{2}}+a\right)\right. \\
\left.\quad-\frac{4 \kappa^{2}}{Q^{2}} a\left[\ln \kappa^{2} \varepsilon^{2}+2 \gamma_{E}+\psi\left(1+\frac{Q^{2}}{4 \kappa^{2}}+a\right)\right]\right\},
\end{aligned}
$$

by means of using the infinite series representation of the digamma function. From the series representation of the $\Gamma$ function it could be derived [38] that

$$
\psi(1+z)=-\gamma_{E}+\sum_{n=1}^{\infty} \frac{z}{n(n+z)},
$$

and that is valid for $z \neq-1,-2, \ldots$. For the particular $\psi$ 's of Eq. (C1) we have

$$
\begin{aligned}
\lim _{Q^{2} \rightarrow \infty} \psi\left(\frac{Q^{2}}{4 \kappa^{2}}+1\right) & =-\gamma_{E}+\sum_{n=0}^{\infty} \frac{1}{n+1} \sum_{k=0}^{\infty}\left(\frac{-M_{V}^{2}(n)}{Q^{2}}\right)^{k} \\
\lim _{Q^{2} \rightarrow \infty} \psi\left(\frac{Q^{2}}{4 \kappa^{2}}+1+\frac{\left(g_{5} R f\right)^{2}}{2 k_{s}}\right) & =-\gamma_{E}+\left(1+\frac{2 \kappa^{2}\left(g_{5} R f\right)^{2}}{k_{s} Q^{2}}\right) \sum_{n=0}^{\infty} \frac{1}{n+1} \sum_{k=0}^{\infty}\left(\frac{-M_{A}^{2}(n)}{Q^{2}}\right)^{k}
\end{aligned}
$$


where for $k=0$ we have $\lim _{N \rightarrow \infty} \sum_{n=1}^{N} \frac{1}{n}=\ln N+\gamma_{E}+\mathcal{O}(1 / N)$.

Substitution of the series expansions yields order by order for $g_{V}^{2} \Pi_{L R}\left(Q^{2}\right) / Q^{2}$,

$$
\begin{gathered}
\left(\frac{1}{Q^{2}}\right)^{0}: \sin ^{2} \theta \frac{R}{2 g_{5}^{2}}\left(\sum_{n=0}^{\infty} \frac{1}{n+1}-\sum_{n=0}^{\infty} \frac{1}{n+1}\right) \\
\left(\frac{1}{Q^{2}}\right)^{1}: 4 \kappa^{2} \sin ^{2} \theta \frac{R}{2 g_{5}^{2}} \sum_{n=0}^{\infty}(1-1)-\sin ^{2} \theta \kappa^{2} a \frac{2 R}{g_{5}^{2}}\left(\ln \varepsilon^{2} \kappa^{2}+\gamma_{E}+\sum_{n=0}^{\infty} \frac{1}{n+1}\right) ; \\
\left(\frac{1}{Q^{2}}\right)^{2}: 4 \kappa^{4} \sin ^{2} \theta a \frac{2 R}{g_{5}^{2}} \sum_{n=0}^{\infty}(1-1) .
\end{gathered}
$$

Considering that 1 and -1 , as well as the fractions in the difference between harmonic sums, appear together for any fixed $n$ we can set these terms to zero (certainly 0 for a finite sum). The remaining at $1 / Q^{2}$ order parentheses cancel due to Eq. (63), when the infinite sum is replaced with the one up to $N_{\max }$. Thus, we show that the terms $1 / Q^{2}$ and $1 / Q^{4}$ are absent as long as $N_{\max }<\infty$.

\section{APPENDIX D: CALCULATIONS RELATED TO THE COUPLINGS OF HIGGS TO EW BOSONS}

We can factorize the misalignment in Eqs. (98) and (99), and come to the following equation:

$$
\begin{aligned}
\mathcal{L}_{\text {eff }} \supset & \frac{g^{2}}{g_{V}^{2}} \frac{\sin 2 \theta}{8 \sqrt{2}} h(q) W_{\mu}^{\alpha}\left(k_{1}\right) W_{\nu}^{\beta}\left(k_{2}\right)\left[\frac{\delta^{2} S_{5 \mathrm{D}}^{(3)}}{\delta \phi_{L \mu}^{\alpha}\left(k_{1}\right) \delta \phi_{b r \nu}^{\beta}\left(k_{2}\right) h(q)}+\frac{\delta^{2} S_{5 \mathrm{D}}^{(3)}}{\delta \phi_{b r \mu}^{\alpha}\left(k_{1}\right) \delta \phi_{L \nu}^{\beta}\left(k_{2}\right) h(q)}\right] \\
& +\frac{g^{2}}{4 g_{V}^{2}} h\left(q_{1}\right) h\left(q_{2}\right) W_{\mu}^{\alpha}\left(k_{1}\right) W_{\nu}^{\beta}\left(k_{2}\right)\left[\cos ^{2} \theta \frac{\delta^{2} S_{5 \mathrm{D}}^{(4)}}{\delta \phi_{L \mu}^{\alpha}\left(k_{1}\right) \delta \phi_{L \nu}^{\beta}\left(k_{2}\right) h\left(q_{1}\right) h\left(q_{2}\right)}\right. \\
& \left.+\frac{\sin ^{2} \theta}{2} \frac{\delta^{2} S_{5 \mathrm{D}}^{(4)}}{\delta \phi_{b r \mu}^{\alpha}\left(k_{1}\right) \delta \phi_{b r \nu}^{\beta}\left(k_{2}\right) h\left(q_{1}\right) h\left(q_{2}\right)}\right] .
\end{aligned}
$$

We have made use of the symmetry of the Lagrangian permitting to substitute $\left\langle h\left|\mathcal{O}_{L \mu}^{\alpha} \mathcal{O}_{b r \nu}^{\beta}\right| 0\right\rangle=-\left\langle h\left|\mathcal{O}_{R \mu}^{\alpha} \mathcal{O}_{b r \nu}^{\beta}\right| 0\right\rangle$ and $\left\langle h h\left|\mathcal{O}_{L \mu}^{\alpha} \mathcal{O}_{L \nu}^{\beta}\right| 0\right\rangle=\left\langle h h\left|\mathcal{O}_{R \mu}^{i} \mathcal{O}_{R \nu}^{\beta}\right| 0\right\rangle=-\left\langle h h\left|\mathcal{O}_{L \mu}^{\alpha} \mathcal{O}_{R \nu}^{\beta}\right| 0\right\rangle$.

Let us explore the triple coupling first. The $5 \mathrm{D}$ action provides two types of contributions

$$
\begin{aligned}
\frac{\delta^{2} S_{5 \mathrm{D}}^{(3)}}{\delta \phi_{L \mu}^{\alpha}\left(k_{1}\right) \delta \phi_{b r \nu}^{\beta}\left(k_{2}\right) h(q)}= & \delta^{\alpha \beta} \eta_{\mu \nu} \frac{R}{g_{5}^{2}}\left(a \kappa^{2} \int d y \frac{e^{-y}}{y} \pi(y) / \chi_{\pi} V\left(k_{1}, y\right) A\left(k_{2}, y\right)\right. \\
& \left.+\frac{1}{4} \int d y \frac{e^{-y}}{y} \partial_{z} \pi(y) / \chi_{\pi} V\left(k_{1}, y\right) \partial_{z} A\left(k_{2}, y\right)\right)
\end{aligned}
$$

and the second variation in (D1) evaluates the same but for exchange $k_{1} \leftrightarrow k_{2}$.

Further, we would like to integrate analytically over $y$. As we substitute the Goldstone profile and the longitudinal vector propagators, all dependence on momenta disappears and the calculation can be performed. For the transverse modes we put the propagators on-shell with $k_{1}^{2}=k_{2}^{2}=M_{W}^{2}$ and consider the limit $M_{W}^{2} \ll 4 \kappa^{2}$. Indeed, we naturally expect the composite resonances to have rather large masses and that limit is substantiated numerically in
Sec. VI. Essentially, we set $k_{1}^{2}=k_{2}^{2}=0$, and the outcome integral is analogous to the expression with the longitudinal propagators.

In the calculation it is convenient to use the definitions in terms of the resonance sums,

$$
\begin{aligned}
A(0, z) & =F \pi(z) / \chi_{\pi}=\Gamma(1+a) \Psi\left(a, 0 ; \kappa^{2} z^{2}\right) \\
& =\sum_{n} \frac{\kappa^{2} z^{2} L_{n}^{1}\left(\kappa^{2} z^{2}\right)}{n+1+a}
\end{aligned}
$$




$$
\partial_{z} A(0, z)=F \partial_{z} \pi(z) / \chi_{\pi}=2 \kappa^{2} z(-a) \Gamma(1+a) \Psi\left(a+1,1 ; \kappa^{2} z^{2}\right)=-2 \kappa^{2} z a \sum_{n} \frac{L_{n}\left(\kappa^{2} z^{2}\right)}{n+1+a} .
$$

Then, the variation (D4) could be estimated quite easily due to the orthogonality of the Laguerre polynomials,

$$
\begin{gathered}
\kappa^{2} a F^{-1} \frac{R}{g_{5}^{2}} \sum_{n_{1}, n_{2}} \frac{\int d y e^{-y} y L_{n_{1}}^{1}(y) L_{n_{2}}^{1}(y)+a \int d y e^{-y} L_{n_{1}}(y) L_{n_{2}}(y)}{\left(n_{1}+a+1\right)\left(n_{2}+a+1\right)} \\
=\frac{1}{2 F} \frac{2 R \kappa^{2} a}{g_{5}^{2}} \sum_{n_{1}, n_{2}} \delta_{n_{1} n_{2}} \frac{n_{1}+1+a}{\left(n_{1}+a+1\right)\left(n_{2}+a+1\right)}=\frac{F}{2} .
\end{gathered}
$$

Here we used for $F^{2}$ the definition of Eq. (68).

We follow the same lines for the quartic couplings. Let us start with the variation in (D2):

$$
\begin{aligned}
\frac{\delta^{2} S_{5 \mathrm{D}}^{(4)}}{\delta \phi_{L \mu}^{\alpha}\left(k_{1}\right) \delta \phi_{L \nu}^{\beta}\left(k_{2}\right) h\left(q_{1}\right) h\left(q_{2}\right)}= & 2 \delta^{\alpha \beta} \eta_{\mu \nu} \frac{R}{4 g_{5}^{2}}\left(a \kappa^{2} \int d y \frac{e^{-y}}{y}\left(\pi(y) / \chi_{\pi}\right)^{2} V\left(k_{1}, y\right) V\left(k_{2}, y\right)\right. \\
& \left.+\frac{1}{4} \int d y \frac{e^{-y}}{y}\left(\partial_{z} \pi(y) / \chi_{\pi}\right)^{2} V\left(k_{1}, y\right) V\left(k_{2}, y\right)\right) \\
= & \frac{1}{4} \delta^{\alpha \beta} \eta_{\mu \nu} F^{-2} \frac{2 R}{g_{5}^{2}} a \kappa^{2} \sum_{n} \frac{n+1+a}{(n+1+a)^{2}}=\frac{1}{4} \delta^{\alpha \beta} \eta_{\mu \nu} .
\end{aligned}
$$

Unfortunately, the situation becomes more involved with the variation over the broken sources in (D3) because the integrals there are quartic in Laguerre polynomials:

$$
\begin{aligned}
\frac{\delta^{2} S_{5 \mathrm{D}}^{(4)}}{\delta \phi_{b r \mu}^{\alpha}\left(k_{1}\right) \delta \phi_{b r \nu}^{\beta}\left(k_{2}\right) h\left(q_{1}\right) h\left(q_{2}\right)}= & \delta^{\alpha \beta} \eta_{\mu \nu} F^{-2} \frac{R}{g_{5}^{2}} a \kappa^{2} \\
& \times \sum_{n_{1}, n_{2}} \frac{\int d y e^{-y} A^{2}(0, y)\left[a / 2 L_{n_{1}}(y) L_{n_{2}}(y)-y L_{n_{1}}^{1}(y) L_{n_{2}}^{1}(y)\right]}{\left(n_{1}+a+1\right)\left(n_{2}+a+1\right)}
\end{aligned}
$$

We can make a calculation at $a=0$, with the result $\frac{\delta^{2} S_{5 \mathrm{D}}^{(4)}}{\delta \phi_{b r \mu}^{\alpha}\left(k_{1}\right) \delta \phi_{b r \nu}^{\beta}\left(k_{2}\right) h\left(q_{1}\right) h\left(q_{2}\right)}=-\frac{1}{2} \delta^{\alpha \beta} \eta_{\mu \nu}$. We extrapolate this estimation to the case of general $a$ when we present the quartic coupling in the effective Lagrangian.

\section{APPENDIX E: CALCULATIONS RELATED TO THE COUPLINGS OF VECTOR RESONANCES TO EW BOSONS}

Here we calculate the relevant three-point functions first. Diagrammatically, we obtain a vertex and three propagators with their residues attached to it. In the body of the paper we report the effective vertex proceeding from connecting two legs to the physical sources and reducing the third one via putting an $n$-th resonance on-shell.

There are not that many different types of three-point functions that can be extracted from Eq. (106);

$$
\begin{aligned}
\left\langle\mathcal{O}_{L \mu_{1}}^{\alpha}\left(q_{1}\right) \mathcal{O}_{L \mu_{2}}^{\beta}\left(q_{2}\right) \mathcal{O}_{L \mu_{3}}^{\gamma}\left(q_{3}\right)\right\rangle & =\left\langle\mathcal{O}_{R \mu_{1}}^{\alpha}\left(q_{1}\right) \mathcal{O}_{R \mu_{2}}^{\beta}\left(q_{2}\right) \mathcal{O}_{R \mu_{3}}^{\gamma}\left(q_{3}\right)\right\rangle \\
& =i \varepsilon^{\alpha \beta \gamma} \operatorname{Lor}_{\mu_{1} \mu_{2} \mu_{3}} \delta\left(q_{1}+q_{2}+q_{3}\right) T_{3 V}\left(q_{1}, q_{2}, q_{3}\right) ; \\
\left\langle\mathcal{O}_{L \mu_{1}}^{\alpha}\left(q_{1}\right) \mathcal{O}_{b r \mu_{2}}^{\beta}\left(q_{2}\right) \mathcal{O}_{b r \mu_{3}}^{\gamma}\left(q_{3}\right)\right\rangle & =\left\langle\mathcal{O}_{R \mu_{1}}^{\alpha}\left(q_{1}\right) \mathcal{O}_{b r \mu_{2}}^{\beta}\left(q_{2}\right) \mathcal{O}_{b r \mu_{3}}^{\gamma}\left(q_{3}\right)\right\rangle \\
& =i \varepsilon^{\alpha \beta \gamma} \operatorname{Lor}_{\mu_{1} \mu_{2} \mu_{3}} \delta\left(q_{1}+q_{2}+q_{3}\right) \frac{1}{2} T_{V 2 A}\left(q_{1}, q_{2}, q_{3}\right) ;
\end{aligned}
$$




$$
\begin{aligned}
\left\langle\mathcal{O}_{b r \mu_{1}}^{4}\left(q_{1}\right) \mathcal{O}_{b r \mu_{2}}^{\alpha}\left(q_{2}\right) \mathcal{O}_{R \mu_{3}}^{\beta}\left(q_{3}\right)\right\rangle & =-\left\langle\mathcal{O}_{b r \mu_{1}}^{4}\left(q_{1}\right) \mathcal{O}_{b r \mu_{2}}^{\alpha}\left(q_{2}\right) \mathcal{O}_{L \mu_{3}}^{\beta}\left(q_{3}\right)\right\rangle \\
& =i \delta^{\alpha \beta} \operatorname{Lor}_{\mu_{1} \mu_{2} \mu_{3}} \delta\left(q_{1}+q_{2}+q_{3}\right) \frac{1}{2} T_{V 2 A}\left(q_{3}, q_{1}, q_{2}\right)
\end{aligned}
$$

There, the Lorentz structure of the correlators is collected into

$$
\operatorname{Lor}_{\mu_{1} \mu_{2} \mu_{3}}\left(q_{1}, q_{2}, q_{3}\right)=\eta_{\mu_{1} \mu_{2}}\left(q_{1}-q_{2}\right)_{\mu_{3}}+\eta_{\mu_{1} \mu_{3}}\left(q_{3}-q_{1}\right)_{\mu_{2}}+\eta_{\mu_{2} \mu_{3}}\left(q_{2}-q_{3}\right)_{\mu_{1}},
$$

and we defined the form factors as follows:

$$
\begin{gathered}
T_{3 V}\left(q_{1}, q_{2}, q_{3}\right)=\frac{R}{g_{5}^{2}} \int d z e^{-\kappa^{2} z^{2}} z^{-1} V\left(q_{1}, z\right) V\left(q_{2}, z\right) V\left(q_{3}, z\right), \\
T_{V 2 A}\left(q_{1}, q_{2}, q_{3}\right)=\frac{R}{g_{5}^{2}} \int d z e^{-\kappa^{2} z^{2}} z^{-1} V\left(q_{1}, z\right) A\left(q_{2}, z\right) A\left(q_{3}, z\right) .
\end{gathered}
$$

Now, to consider the possible interactions with $W$ and $B$ bosons we write down the relevant three-point functions:

$$
\begin{aligned}
& \left\langle\mathcal{O}_{L / R \mu_{1}}^{\alpha}\left(q_{1}\right) \tilde{J}_{L \mu_{2}}^{\beta}\left(q_{2}\right) \tilde{J}_{L \mu_{3}}^{\gamma}\left(q_{3}\right)\right\rangle=\frac{g^{2}}{8 g_{V}^{2}} i \varepsilon^{\alpha \beta \gamma} \operatorname{Lor}_{\mu_{1} \mu_{2} \mu_{3}}\left(q_{1}, q_{2}, q_{3}\right) \delta\left(q_{1}+q_{2}+q_{3}\right) \\
& \times\left[(1 \pm \cos \theta)^{2} T_{3 V}\left(q_{1}, q_{2}, q_{3}\right)+\sin ^{2} \theta T_{V 2 A}\left(q_{1}, q_{2}, q_{3}\right)\right] ; \\
& \left\langle\mathcal{O}_{L / R \mu_{1}}^{\alpha}\left(q_{1}\right) \tilde{J}_{L \mu_{2}}^{\beta}\left(q_{2}\right) \tilde{J}_{R \mu_{3}}^{3}\left(q_{3}\right)\right\rangle=\left\langle\mathcal{O}_{L / R \mu_{1}}^{\alpha}\left(q_{1}\right) \tilde{J}_{R \mu_{2}}^{3}\left(q_{2}\right) \tilde{J}_{L \mu_{3}}^{\beta}\left(q_{3}\right)\right\rangle \\
& =\frac{g g^{\prime}}{8 g_{V}^{2}} i \varepsilon^{\alpha \beta 3} \operatorname{Lor}_{\mu_{1} \mu_{2} \mu_{3}}\left(q_{1}, q_{2}, q_{3}\right) \delta\left(q_{1}+q_{2}+q_{3}\right) \\
& \times\left[\left(1-\cos ^{2} \theta\right) T_{3 V}\left(q_{1}, q_{2}, q_{3}\right)-\sin ^{2} \theta T_{V 2 A}\left(q_{1}, q_{2}, q_{3}\right)\right] ; \\
& \left\langle\mathcal{O}_{b r \mu_{1}}^{\alpha}\left(q_{1}\right) \tilde{J}_{L \mu_{2}}^{\beta}\left(q_{2}\right) \tilde{J}_{L \mu_{3}}^{\gamma}\left(q_{3}\right)\right\rangle=-\frac{g^{2}}{2 g_{V}^{2}} \frac{\sin \theta}{\sqrt{2}} i \varepsilon^{\alpha \beta \gamma} \operatorname{Lor}_{\mu_{1} \mu_{2} \mu_{3}}\left(q_{1}, q_{2}, q_{3}\right) \delta\left(q_{1}+q_{2}+q_{3}\right) \\
& \times\left[T_{V 2 A}\left(q_{2}, q_{1}, q_{3}\right)+T_{V 2 A}\left(q_{3}, q_{2}, q_{1}\right)\right] \text {; } \\
& \left\langle\mathcal{O}_{b r \mu_{1}}^{\alpha}\left(q_{1}\right) \tilde{J}_{L \mu_{2}}^{\beta}\left(q_{2}\right) \tilde{J}_{R \mu_{3}}^{3}\left(q_{3}\right)\right\rangle=\frac{g g^{\prime}}{2 g_{V}^{2}} \frac{\sin \theta}{\sqrt{2}} i \varepsilon^{\alpha \beta 3} \operatorname{Lor}_{\mu_{1} \mu_{2} \mu_{3}}\left(q_{1}, q_{2}, q_{3}\right) \delta\left(q_{1}+q_{2}+q_{3}\right) \\
& \times\left[T_{V 2 A}\left(q_{2}, q_{1}, q_{3}\right)-T_{V 2 A}\left(q_{3}, q_{2}, q_{1}\right)\right] ; \\
& \left\langle\mathcal{O}_{b r \mu_{1}}^{4}\left(q_{1}\right) \tilde{J}_{L \mu_{2}}^{\alpha}\left(q_{2}\right) \tilde{J}_{L \mu_{3}}^{\beta}\left(q_{3}\right)\right\rangle=\frac{g^{2} \sin 2 \theta}{8 \sqrt{2} g_{V}^{2}} \delta^{\alpha \beta} \operatorname{Lor}_{\mu_{1} \mu_{2} \mu_{3}}\left(q_{1}, q_{2}, q_{3}\right) \delta\left(q_{1}+q_{2}+q_{3}\right) \\
& \times\left[T_{V 2 A}\left(q_{3}, q_{1}, q_{2}\right)-T_{V 2 A}\left(q_{2}, q_{1}, q_{3}\right)\right] ; \\
& \left\langle\mathcal{O}_{b r \mu_{1}}^{4}\left(q_{1}\right) \tilde{J}_{R \mu_{2}}^{3}\left(q_{2}\right) \tilde{J}_{R \mu_{3}}^{3}\left(q_{3}\right)\right\rangle=\frac{g^{\prime 2} \sin 2 \theta}{8 \sqrt{2} g_{V}^{2}} \operatorname{Lor}_{\mu_{1} \mu_{2} \mu_{3}}\left(q_{1}, q_{2}, q_{3}\right) \delta\left(q_{1}+q_{2}+q_{3}\right) \\
& \times\left[T_{V 2 A}\left(q_{3}, q_{1}, q_{2}\right)-T_{V 2 A}\left(q_{2}, q_{1}, q_{3}\right)\right] \text {; } \\
& \left\langle\mathcal{O}_{b r \mu_{1}}^{4}\left(q_{1}\right) \tilde{J}_{L \mu_{2}}^{3}\left(q_{2}\right) \tilde{J}_{R \mu_{3}}^{3}\left(q_{3}\right)\right\rangle=\frac{g g^{\prime} \sin 2 \theta}{8 \sqrt{2} g_{V}^{2}} \operatorname{Lor}_{\mu_{1} \mu_{2} \mu_{3}}\left(q_{1}, q_{2}, q_{3}\right) \delta\left(q_{1}+q_{2}+q_{3}\right) \\
& \times\left[T_{V 2 A}\left(q_{3}, q_{1}, q_{2}\right)-T_{V 2 A}\left(q_{2}, q_{1}, q_{3}\right)\right] .
\end{aligned}
$$

Only a few $B B$-resonance interactions are possible due to the epsilon-tensor on the right-hand side of the holographic threepoint functions. 
Further, we reduce the leg corresponding to $q_{1}$ momentum and consider the limit $q_{2,3}^{2} \ll 4 \kappa^{2}$ for other two momenta. For the $n$-th excitation of the left/right resonances in the unbroken sector, this means

$$
\begin{gathered}
T_{3 V}\left(q_{1}, q_{2}, q_{3}\right) \rightarrow \sqrt{\frac{R}{2 g_{5}^{2}(n+1)}} \int d y e^{-y} L_{n}^{1}(y)=\sqrt{\frac{R}{2 g_{5}^{2}(n+1)},} \\
T_{V 2 A}\left(q_{1}, q_{2}, q_{3}\right) \rightarrow \sqrt{\frac{R}{2 g_{5}^{2}(n+1)}} \int d y e^{-y} L_{n}^{1}(y) \Gamma^{2}(1+a) \Psi^{2}(a, 0 ; y),
\end{gathered}
$$

where the latter integral can be calculated for a given $n$. For $n=0: 1-2 a+2 a^{2} \psi_{1}(1+a)$.

For the $n$-th excitation of the resonances from the broken sector one of the broken legs should be reduced, and we get

$$
\begin{aligned}
T_{V 2 A}\left(q_{2}, q_{1}, q_{3}\right) \quad \text { or } \quad T_{V 2 A}\left(q_{3}, q_{2}, q_{1}\right) & \rightarrow \sqrt{\frac{R}{2 g_{5}^{2}(n+1)}} \sum_{n^{\prime}} \frac{\int d y e^{-y} L_{n}^{1}(y) L_{n^{\prime}}^{1}(y)}{n^{\prime}+1+a} \\
& =\sqrt{\frac{R(n+1)}{2 g_{5}^{2}} \frac{1}{n+1+a}} .
\end{aligned}
$$

Some triple couplings will not be included in the effective Lagrangian. These are: $A_{b r}^{4} W^{\alpha} W^{\alpha}, A_{b r}^{4} B B, A_{b r}^{4} W^{3} B, A_{b r}^{\alpha} W^{\beta} B$. The reason for this is that in the corresponding three-point functions the leading term in the limit $q_{2,3}^{2} \ll 4 \kappa^{2}$ is zero due to the subtraction of the form factors. The first contribution is $\sim \frac{M_{W}^{2}}{4 \kappa^{2}}$ and, thus, is strongly suppressed. We abstain from considering observables of this order in this work.

[1] J. Maldacena, Int. J. Theor. Phys. 38, 1113 (1999).

[2] S. Gubser, I. Klebanov, and A. Polyakov, Phys. Lett. B 428, 105 (1998).

[3] E. Witten, Adv. Theor. Math. Phys. 2, 253 (1998).

[4] J. Erlich, E. Katz, D. T. Son, and M. A. Stephanov, Phys. Rev. Lett. 95, 261602 (2005).

[5] L. Da Rold and A. Pomarol, Nucl. Phys. B721, 79 (2005).

[6] A. Karch, E. Katz, D. T. Son, and M. A. Stephanov, Phys. Rev. D 74, 015005 (2006).

[7] D. B. Kaplan and H. Georgi, Phys. Lett. 136B, 183 (1984); D. B. Kaplan, H. Georgi, and S. Dimopoulos, Phys. Lett. 136B, 187 (1984); H. Georgi, D. B. Kaplan, and P. Galison, Phys. Lett. 143B, 152 (1984); H. Georgi and D. B. Kaplan, Phys. Lett. 145B, 216 (1984); M. J. Dugan, H. Georgi, and D. B. Kaplan, Nucl. Phys. B254, 299 (1985).

[8] K. Agashe, R. Contino, and A. Pomarol, Nucl. Phys. B719, 165 (2005).

[9] K. Agashe and R. Contino, Nucl. Phys. B742, 59 (2006).

[10] A. D. Medina, N. R. Shah, and C. E. M. Wagner, Phys. Rev. D 76, 095010 (2007).

[11] R. Contino, Y. Nomura, and A. Pomarol, Nucl. Phys. B671, 148 (2003).

[12] G. Panico, M. Serone, and A. Wulzer, Nucl. Phys. B739, 186 (2006).

[13] M. Serone, New J. Phys. 12, 075013 (2010).
[14] G. Panico, M. Safari, and M. Serone, J. High Energy Phys. 02 (2011) 103.

[15] Y. Hosotani, Phys. Lett. 126B, 309 (1983).

[16] A. Falkowski and M. Perez-Victoria, J. High Energy Phys. 08 (2008) 107.

[17] S. Gasiorowicz and D. A. Geffen, Rev. Mod. Phys. 41, 531 (1969); A. H. Fariborz, R. Jora, and J. Schechter, Phys. Rev. D 72, 034001 (2005); D. Parganlija, F. Giacosa, and D. H. Rischke, Phys. Rev. D 82, 054024 (2010).

[18] J. Hirn and V. Sanz, J. High Energy Phys. 12 (2005) 030.

[19] J. Hirn, N. Rius, and V. Sanz, Phys. Rev. D 73, 085005 (2006).

[20] L. Cappiello, G. D’Ambrosio, and D. Greynat, Eur. Phys. J. C 75, 465 (2015).

[21] D. Espriu and A. Katanaeva, Phys. Rev. D 101, 074017 (2020).

[22] B. Bellazzini, C. Csáki, and J. Serra, Eur. Phys. J. C 74, 2766 (2014).

[23] G. Panico and A. Wulzer, Lect. Notes Phys., arXiv: $1506.01961 \mathrm{v} 2$.

[24] G. Ferretti and D. Karateev, J. High Energy Phys. 03 (2014) 077.

[25] G. Cacciapaglia and F. Sannino, J. High Energy Phys. 04 (2014) 111.

[26] O. Aharony, S. S. Gubser, J. M. Maldacena, H. Ooguri, and Y. Oz, Phys. Rep. 323, 183 (2000). 
[27] D. Z. Freedman, S. D. Mathur, A. Matusis, and L. Rastelli, Nucl. Phys. B546, 96 (1999).

[28] L. Reinders, H. Rubinstein, and S. Yazaki, Phys. Rep. 127, 1 (1985).

[29] S. S. Afonin and D. Espriu, J. High Energy Phys. 06 (2006) 047.

[30] G. Altarelli and R. Barbieri, Phys. Lett. B 253, 161 (1991).

[31] M.E. Peskin and T. Takeuchi, Phys. Rev. D 46, 381 (1992).

[32] S. Weinberg, Phys. Rev. Lett. 18, 507 (1967).

[33] P. A. Zyla et al. (Particle Data Group), Prog. Theor. Exp. Phys. 2020, 083C01 (2020).
[34] G. Aad et al. (The ATLAS Collaboration), J. High Energy Phys. 11 (2015) 206.

[35] G. Aad et al. (ATLAS Collaboration), Phys. Rev. D 101, 012002 (2020).

[36] A. M. Sirunyan et al. (CMS Collaboration), Eur. Phys. J. C 79, 421 (2019).

[37] R. Delgado, A. Dobado, D. Espriu, C. Garcia-Garcia, M. Herrero, X. Marcano, and J. Sanz-Cillero, J. High Energy Phys. 11 (2017) 098.

[38] Higher Transcendental Functions (Bateman Manuscript Project), edited by A. Erdélyi, Vol. 1 (McGraw-Hill, New York, 1953). 